FTUAM 07/02

IFT-UAM/CSIC-07-09

KUNS-2091

arXiv:yymm.nnnn

6 September 2007

\title{
Prospects for the direct detection of neutralino dark matter in orbifold scenarios
}

\author{
David G. Cerdeño ${ }^{a, b}$, Tatsuo Kobayashi ${ }^{c}$, Carlos Muñoz $^{a, b}$ \\ ${ }^{a}$ Departamento de Física Teórica C-XI, Universidad Autónoma de Madrid, \\ Cantoblanco, E-28049 Madrid, Spain \\ ${ }^{b}$ Instituto de Física Teórica C-XVI, Universidad Autónoma de Madrid, \\ Cantoblanco, E-28049 Madrid, Spain \\ ${ }^{c}$ Department of Physics, Kyoto University, Kyoto 606-8502, Japan.
}

\begin{abstract}
We analyse the phenomenology of orbifold scenarios from the heterotic superstring, and the resulting theoretical predictions for the direct detection of neutralino dark matter. In particular, we study the parameter space of these constructions, computing the low-energy spectrum and taking into account the most recent experimental and astrophysical constraints, as well as imposing the absence of dangerous charge and colour breaking minima. In the remaining allowed regions the spin-independent part of the neutralino-proton cross section is calculated and compared with the sensitivity of dark matter detectors. In addition to the usual non universalities of the soft terms in orbifold scenarios due to the modular weight dependence, we also consider D-term contributions to scalar masses. These are generated by the presence of an anomalous $U(1)$, providing more flexibility in the resulting soft terms, and are crucial in order to avoid charge and colour breaking minima. Thanks to the D-term contribution, large neutralino detection cross sections can be found, within the reach of projected dark matter detectors.
\end{abstract}

PACS: 11.25.Wx, 95.35.+d

Key words: String phenomenology, Dark matter 


\section{Introduction}

One of the most interesting candidates for the dark matter in the Universe is a Weakly Interacting Massive Particle (WIMP), and in fact many underground experiments are being carried out around the world in order to detect its flux on the Earth [1]. These try to observe the elastic scattering of WIMPs on target nuclei through nuclear recoils. Although one of the experiments, the DAMA collaboration [2], reported data favouring the existence of a signal with WIMP-proton cross section $\approx 10^{-6}-10^{-5}$ pb for a WIMP mass smaller than $500-900 \mathrm{GeV}[2,3]$, other collaborations such as CDMS Soudan [4], EDELWEISS [5], and ZEPLIN I [6] claim to have excluded important regions of the DAMA parameter space ${ }^{1}$. Recently, the XENON10 experiment at the Gran Sasso National Laboratory [8] has set the strongest upper limit for the WIMP-proton cross section, further disfavouring the DAMA result. This controversy will be solved in the future since many experiments are running or in preparation around the world. For example, LIBRA [9] and ANAIS [10] will probe the region compatible with DAMA result. Moreover, CDMS Soudan will be able to explore a WIMP-proton cross section $\sigma \gtrsim 2 \times 10^{-8} \mathrm{pb}$, and planned 1 tonne Ge/Xe detectors are expected to reach cross sections as low as $10^{-10} \mathrm{pb}[11]$.

The leading candidate within the class of WIMPs is the lightest neutralino, $\tilde{\chi}_{1}^{0}$, a particle predicted by supersymmetric (SUSY) extensions of the standard model. Given the experimental situation, and assuming that the dark matter is a neutralino, it is natural to wonder how big the cross section for its direct detection can be. This analysis is crucial in order to know the possibility of detecting dark matter in the experiments. In fact, the analysis of the neutralino-proton cross section has been carried out by many authors and during many years [1]. The most recent studies take into account the present experimental and astrophysical constraints on the parameter space. Concerning the former, the lower bound on the Higgs mass, the $b \rightarrow s \gamma$ and $B_{s}^{0} \rightarrow \mu^{+} \mu^{-}$branching ratios, and the muon anomalous magnetic moment have been considered. The astrophysical bounds on the dark matter density, $0.1 \lesssim \Omega h^{2} \lesssim 0.3$ $\left(0.095 \lesssim \Omega h^{2} \lesssim 0.112\right.$ if we take into account the recent data obtained by the WMAP satellite [12]), have also been imposed on the theoretical computation of the relic neutralino density, assuming thermal production. In addition, the constraints that the absence of dangerous charge and colour breaking minima imposes on the parameter space have also been implemented [13].

\footnotetext{
${ }^{1}$ For attempts to show that DAMA and these experiments might not be in conflict, see Ref. [7].
} 
In the usual minimal supergravity (mSUGRA) scenario, where the soft terms of the minimal supersymmetric standard model (MSSM) are assumed to be universal at the unification scale, $M_{G U T} \approx 2 \times 10^{16} \mathrm{GeV}$, and radiative electroweak symmetry breaking is imposed, the neutralino-proton cross section turns out to be constrained by $\sigma_{\tilde{\chi}_{1}^{0}-p} \lesssim 3 \times 10^{-8} \mathrm{pb}$. Clearly, in this case, present experiments are not sufficient and only the planned 1 tonne Ge/Xe detectors would be able to test part of the parameter space. However, in the presence of non-universal soft scalar and gaugino masses [14] the cross section can be increased significantly [15] in some regions with respect to the universal scenario (see, e.g., the discussion in [16], and references therein). Although the current upper limit on the decay $B_{s} \rightarrow \mu^{+} \mu^{-}$seriously affects these results, as was pointed out in [17], regions of the parameter space can still be found where the neutralino detection cross section can be within the reach of experiments such as CDMS Soudan. An analysis, summarizing all these results in the context of SUGRA, can be found in $[18]$.

On the other hand, the low-energy limit of superstring theory is SUGRA, and therefore the neutralino is also a candidate for dark matter in superstring constructions. Let us recall that, in the late eighties, working in the context of the $E_{8} \times E_{8}$ heterotic superstring, a number of interesting four-dimensional vacua with particle content not far from that of the SUSY standard model were found (see, e.g., the discussion in the introduction of [19], and references therein). Such constructions have a natural hidden sector built-in: the complex dilaton field $S$ arising from the gravitational sector of the theory, and the complex moduli fields $T_{i}$ parametrizing the size and shape of the compactified space. The auxiliary fields of those gauge singlets can be the seed of SUSY breaking, solving the arbitrariness of SUGRA where the hidden sector is not constrained. In addition, in superstrings the gauge kinetic function, $f_{a}\left(S, T_{i}\right)$, and the Kähler potential, $K\left(S, S^{*}, T_{i}, T_{i}^{*}\right)$, can be computed explicitly, leading to interesting predictions for the soft parameters [20]. More specifically, in orbifold constructions they show a lack of universality due to the modular weight dependence. From these resulting SUGRA models one can also obtain predictions for the value of the neutralino-proton cross section. In fact, analyses of the detection cross section in these constructions were carried out in the past in [21-23].

Our aim in this work is to study in detail the phenomenology of these orbifold models, including the most recent experimental constraints on low-energy observables, as well as those coming from charge and colour breaking minima, and to determine how large the cross section for the direct detection of neutralino dark matter can be. 
We therefore calculate the theoretical predictions for the spin-independent part of the neutralino-nucleon cross section, $\sigma_{\tilde{\chi}_{1}^{0}-p}$, and compare it with the sensitivities of present and projected experiments. Since the soft terms in superstring scenarios are a subset of the general soft terms studied in SUGRA theories we make use of previous results on departures from the mSUGRA scenario to look for values of the orbifold soft terms giving rise to a large cross section accessible for experiments.

In addition, we introduce a new ingredient in the analysis, namely the modification produced in the soft parameters by the presence of an anomalous $U(1)$. Let us recall that in string theory, and in particular in orbifold constructions [24, 25] of the heterotic superstring [26], the gauge groups obtained after compactification are larger than the standard model gauge group, and contain generically extra $U(1)$ symmetries, $S U(3) \times$ $S U(2) \times U(1)^{n}[27]$. One of these $U(1)$ 's is usually anomalous, and although its anomaly is cancelled by the four-dimensional Green-Schwarz (GS) mechanism, it generates a Fayet-Iliopoulos (FI) contribution to the D-term [28]. This effect is crucial for model building [29] since some scalars acquire large vacuum expectation values (VEVs) in order to cancel the FI contribution, thereby breaking the extra gauge symmetries, and allowing the construction of realistic standard-like models in the context of the $Z_{3}$ orbifold [30-32] (see also [33]). Recently other interesting models in the context of the $Z_{6}$ orbifold [34-36], and $Z_{12}$ orbifold [37,38], have been analysed. Due to the FI breaking, also D-term contributions to the soft scalar masses are generated [39-44]. This allows more flexibility in the soft terms and, consequently, in the computation of the associated neutralino-proton cross section.

The paper is organised as follows. In Section 2 we briefly review the departures from mSUGRA which give rise to large values of the neutralino detection cross section. Then, in the next sections, we use this analysis to study several orbifold scenarios where such departures may be present. Special emphasis is put on the effect of the various experimental constraints on the SUSY spectrum and low-energy observables. We start in Section 3 with the simplest (but not the most common) possibility, where an anomalous $U(1)$ is not present. Then, in Section 4, we discuss the important modifications produced in the soft terms by the presence of an anomalous $U(1)$, and their effects on the computation of the neutralino-proton cross section, considering the effect of D-term contributions to soft scalar masses. The conclusions are left for Section 5 . 


\section{Neutralino-proton cross section and departures from mSUGRA}

In this section we review possible departures from the mSUGRA scenario, and their impact on the neutralino-proton cross section. This will allow us to discuss orbifold scenarios more easily. Let us first recall that in mSUGRA one has only four free parameters defined at the GUT scale: the soft scalar mass, $m$, the soft gaugino mass, $M$, the soft trilinear coupling, $A$, and the ratio of the Higgs vacuum expectation values, $\tan \beta \equiv\left\langle H_{u}^{0}\right\rangle /\left\langle H_{d}^{0}\right\rangle$. In addition, the sign of the Higgsino mass parameter, $\mu$, remains undetermined. Using these inputs the neutralino-proton cross section has been analysed exhaustively in the literature, as mentioned in the Introduction. Taking into account all kind of experimental and astrophysical constraints, the resulting scalar cross section is bounded to be $\sigma_{\tilde{\chi}_{1}^{0}-p} \lesssim 3 \times 10^{-8} \mathrm{pb}$.

Departures from the universal structure of the soft parameters in mSUGRA allow to increase the neutralino-proton cross section significantly. As it was shown in the literature, it is possible to enhance the scattering channels involving exchange of CPeven neutral Higgses by reducing the Higgs masses, and also by increasing the Higgsino components of the lightest neutralino. A brief analysis based on the Higgs mass parameters, $m_{H_{d}}^{2}$ and $m_{H_{u}}^{2}$, at the electroweak scale can clearly show how these effects can be achieved.

First, a decrease in the values of the Higgs masses can be obtained by increasing $m_{H_{u}}^{2}$ at the electroweak scale (i.e., making it less negative) and/or decreasing $m_{H_{d}}^{2}$. More specifically, the value of the mass of the heaviest CP-even Higgs, $H$, can be very efficiently lowered under these circumstances. This is easily understood by analysing the (tree-level) mass of the CP-odd Higgs $A$, which for reasonably large values of $\tan \beta$ can be approximated as $m_{A}^{2} \approx m_{H_{d}}^{2}-m_{H_{u}}^{2}-M_{Z}^{2}$. Since the heaviest CP-even Higgs, $H$, is almost degenerate in mass with $A$, lowering $m_{A}^{2}$ we obtain a decrease in $m_{H}^{2}$ which leads to an increase in the scattering channels through Higgs exchange

Second, through the increase in the value of $m_{H_{u}}^{2}$ an increase in the Higgsino components of the lightest neutralino can also be achieved. Making $m_{H_{u}}^{2}$ less negative, its positive contribution to $\mu^{2}$ in the minimization of the Higgs potential would be smaller. Eventually $|\mu|$ will be of the order of $M_{1}, M_{2}$ and $\tilde{\chi}_{1}^{0}$ will then be a mixed Higgsinogaugino state. Thus scattering channels through Higgs exchange become more important than in mSUGRA, where $|\mu|$ is large and $\tilde{\chi}_{1}^{0}$ is mainly bino. It is worth emphasizing 
however that the effect of lowering the Higgs masses is typically more important, since it can provide large values for the neutralino-nucleon cross section even in the case of bino-like neutralinos.

Non-universal soft parameters can produce the above mentioned effects. Let us consider in particular the non-universality in the scalar masses, which will be the most interesting possibility in orbifold scenarios. We can parametrize these in the Higgs sector, at the high-energy scale, as follows:

$$
m_{H_{d}}^{2}=m^{2}\left(1+\delta_{H_{d}}\right), \quad m_{H_{u}}^{2}=m^{2}\left(1+\delta_{H_{u}}\right) .
$$

Concerning squarks and sleptons we will assume that the three generations have the same mass structure:

$$
\begin{aligned}
& m_{Q_{L}}^{2}=m^{2}\left(1+\delta_{Q_{L}}\right), \quad m_{u_{R}}^{2}=m^{2}\left(1+\delta_{u_{R}}\right) \\
& m_{e_{R}}^{2}=m^{2}\left(1+\delta_{e_{R}}\right), \quad m_{d_{R}}^{2}=m^{2}\left(1+\delta_{d_{R}}\right) \\
& m_{L_{L}}^{2}=m^{2}\left(1+\delta_{L_{L}}\right) .
\end{aligned}
$$

Such a structure avoids potential problems with flavour changing neutral currents ${ }^{2}$ (FCNC), and arises naturally e.g. in $Z_{3}$ orbifold constructions with two Wilson lines, where realistic models have been obtained. Note also that whereas all $\delta$ 's in (2.2) have to satisfy $\delta \geq-1$ in order to avoid an unbounded from below (UFB) direction breaking charge and colour ${ }^{3}, \delta_{H_{u, d}} \leq-1$ in (2.1) is possible as long as $m_{1}^{2}=m_{H_{d}}^{2}+\mu^{2}>0$ and $m_{2}^{2}=m_{H_{u}}^{2}+\mu^{2}>0$ are fulfilled.

An increase in $m_{H_{u}}^{2}$ at the electroweak scale can be obviously achieved by increasing its value at the high-energy scale, i.e., with the choice $\delta_{H_{u}}>0$. In addition, this is also produced when $m_{Q_{L}}^{2}$ and $m_{u_{R}}^{2}$ at the high-energy scale decrease, i.e. taking $\delta_{Q_{L}, u_{R}}<0$, due to their (negative) contribution proportional to the top Yukawa coupling in the renormalization group equation (RGE) of $m_{H_{u}}^{2}$.

Similarly, a decrease in the value of $m_{H_{d}}^{2}$ at the electroweak scale can be obtained by decreasing it at the high-energy scale with $\delta_{H_{d}}<0$. The same effect is obtained when $m_{Q_{L}}^{2}$ and $m_{d_{R}}^{2}$ increase at the high-energy scale, due to their (negative) contribution

\footnotetext{
${ }^{2}$ Another possibility would be to assume that the first two generations have the common scalar mass $m$, and that non-universalities are allowed only for the third generation (as it occurs for the models analysed in Ref. [45]). This would not modify our analysis since, as we will see below, only the third generation is relevant in our discussion.

${ }^{3}$ If we allow metastability of our vacuum, tachyonic masses for some sfermions, $\delta<-1$, at the high-energy scale might be allowed. However, we do not consider such a possibility.
} 
proportional to the bottom Yukawa coupling in the RGE of $m_{H_{d}}^{2}$. Thus one can deduce that $m_{A}^{2}$ will also be reduced by choosing $\delta_{Q_{L}, d_{R}}>0$.

In fact non-universality in the Higgs sector gives the most important effect, and including the one in the sfermion sector the cross section only increases slightly.

Taking into account this analysis, several scenarios were discussed in Ref. [13], obtaining that large values for the cross section are possible. For example, with $\delta_{H_{d}}=$ $0, \delta_{H_{u}}=1 ; \delta_{H_{d}}=-1, \delta_{H_{u}}=0 ; \delta_{H_{d}}=-1, \delta_{H_{u}}=1$, regions of the parameter space are found which are accessible for experiments such as CDMS Soudan [18]. Interestingly, it was also realised that these choices of parameters were helpful in order to prevent the appearance of UFB minima in the Higgs potential.

The different UFB directions were classified in Ref. [46]. Among these, the one labelled as UFB-3, which involves VEVs for the fields $\left\{H_{u}, \tilde{\nu}_{L_{i}}, \tilde{e}_{L_{j}}, \tilde{e}_{R_{j}}\right\}$ with $i \neq j$, yields the strongest bound. After an analytical minimization of the relevant terms of the scalar potential the value of the $\tilde{\nu}_{L_{i}}, \tilde{e}_{L_{j}}, \tilde{e}_{R_{j}}$ fields can be written in terms of $H_{u}$. Then, for any value of $\left|H_{u}\right|<M_{G U T}$ satisfying

$$
\left|H_{u}\right|>\sqrt{\frac{\mu^{2}}{4 \lambda_{e_{j}}^{2}}+\frac{4 m_{L_{i}}^{2}}{g^{\prime 2}+g_{2}^{2}}}-\frac{|\mu|}{2 \lambda_{e_{j}}},
$$

the potential along the UFB-3 direction reads

$$
V_{\mathrm{UFB}-3}=\left(m_{H_{u}}^{2}+m_{L_{i}}^{2}\right)\left|H_{u}\right|^{2}+\frac{|\mu|}{\lambda_{e_{j}}}\left(m_{L_{j}}^{2}+m_{e_{j}}^{2}+m_{L_{i}}^{2}\right)\left|H_{u}\right|-\frac{2 m_{L_{i}}^{4}}{g^{\prime 2}+g_{2}^{2}} .
$$

Otherwise

$$
V_{\mathrm{UFB}-3}=m_{H_{u}}^{2}\left|H_{u}\right|^{2}+\frac{|\mu|}{\lambda_{e_{j}}}\left(m_{L_{j}}^{2}+m_{e_{j}}^{2}\right)\left|H_{u}\right|+\frac{1}{8}\left(g^{\prime 2}+g_{2}^{2}\right)\left[\left|H_{u}\right|^{2}+\frac{|\mu|}{\lambda_{e_{j}}}\left|H_{u}\right|\right]^{2} .
$$

In these expressions $\lambda_{e_{j}}$ denotes the leptonic Yukawa coupling of the $j$ th generation, the deepest direction corresponding to $e_{j}=\tau$. The UFB-3 condition is then $V_{\mathrm{UFB}-3}(Q=$ $\hat{Q})>V_{\text {real min. }}\left(Q=M_{S U S Y}\right)$, where $V_{\text {real min. }}=-\frac{1}{8}\left(g^{\prime 2}+g_{2}^{2}\right)\left(v_{u}^{2}-v_{d}^{2}\right)^{2}$, with $v_{u, d}=$ $\left\langle H_{u, d}\right\rangle$, is the value of the potential at the realistic minimum. $V_{\text {real min }}$ is evaluated at the typical scale of SUSY masses, $M_{S U S Y}$, and $V_{\mathrm{UFB}-3}$ at the renormalization scale, $\hat{Q}$, which is chosen to be $\hat{Q} \sim \operatorname{Max}\left(\lambda_{\text {top }}\left|\mathrm{H}_{\mathrm{u}}\right|, \mathrm{M}_{\text {SUSY }}\right)$, in order to minimize the effect of one-loop corrections to the scalar potential.

As we see from Eqs. (2.4) and (2.5), the potential along this direction can be lifted when $m_{H_{u}}^{2}$ increases (becomes less negative) and for large values of the stau mass 
parameters, thereby making the UFB-3 condition less restrictive. In this sense, nonuniversal soft terms, like the ones discussed above, can be very helpful.

The question now is whether it is possible to find explicit realisations of these scenarios within orbifold models. In the following sections we will study this issue in detail.

\section{Orbifold scenarios}

Let us recall first the structure of the SUGRA theory in four-dimensional constructions from the heterotic superstring. The tree-level gauge kinetic function is independent of the moduli sector and is simply given by

$$
f_{a}=k_{a} S
$$

where $k_{a}$ is the Kac-Moody level of the gauge factor. Usually (level one case) one takes $k_{3}=k_{2}=\frac{3}{5} k_{1}=1$ for the MSSM. In any case, the values $k_{a}$ are irrelevant for the tree-level computation since they do not contribute to the soft parameters. On the other hand, the Kähler potential has been computed for six-dimensional Abelian orbifolds, where three moduli $T_{i}$ are generically present. For this class of models the Kähler potential has the form

$$
K=-\log \left(S+S^{*}\right)-\sum_{i} \log \left(T_{i}+T_{i}^{*}\right)+\sum_{\alpha}\left|C^{\alpha}\right|^{2} \Pi_{i}\left(T_{i}+T_{i}^{*}\right)^{n_{\alpha}^{i}} .
$$

Here $n_{\alpha}^{i}$ are (zero or negative) fractional numbers usually called 'modular weights' of the matter fields $C^{\alpha}$.

In order to determine the pattern of soft parameters it is crucial to know which fields, either $S$ or $T_{i}$, play the predominant role in the process of SUSY breaking. Thus one can introduce a parametrization for the VEVs of dilaton and moduli auxiliary fields [47]. A convenient one is given by [47-49]

$$
\begin{aligned}
F^{S} & =\sqrt{3}\left(S+S^{*}\right) m_{3 / 2} \sin \theta, \\
F^{i} & =\sqrt{3}\left(T_{i}+T_{i}^{*}\right) m_{3 / 2} \cos \theta \Theta_{i},
\end{aligned}
$$

where $i=1,2,3$ labels the three complex compact dimensions, $m_{3 / 2}$ is the gravitino mass, and the angles $\theta$ and $\Theta_{i}$, with $\sum_{i}\left|\Theta_{i}\right|^{2}=1$, parametrize the Goldstino direction in the $S, T_{i}$ field space. Here we are neglecting phases and the cosmological constant vanishes by construction. 
Using this parametrization and Eqs. (3.6) and (3.7) one obtains the following results for the soft terms [47-49]:

$$
\begin{aligned}
M_{a}= & \sqrt{3} m_{3 / 2} \sin \theta \\
m_{\alpha}^{2}= & m_{3 / 2}^{2}\left(1+3 \cos ^{2} \theta \sum_{i} n_{\alpha}^{i} \Theta_{i}^{2}\right), \\
A_{\alpha \beta \gamma}= & -\sqrt{3} m_{3 / 2}(\sin \theta \\
& \left.+\cos \theta \sum_{i} \Theta_{i}\left[1+n_{\alpha}^{i}+n_{\beta}^{i}+n_{\gamma}^{i}-\left(T_{i}+T_{i}^{*}\right) \partial_{i} \log \lambda_{\alpha \beta \gamma}\right]\right) .
\end{aligned}
$$

Although in the case of the $A$ parameter an explicit $T_{i}$-dependence may appear in the term proportional to $\partial_{i} \log \lambda_{\alpha \beta \gamma}$, where $\lambda_{\alpha \beta \gamma}\left(T_{i}\right)$ are the Yukawa couplings and $\partial_{i} \equiv \partial / \partial_{T_{i}}$, it disappears in several interesting cases $[47,49]$. For example, the $A$ term which is relevant to electroweak symmetry-breaking is the one associated to the top-quark Yukawa coupling. Thus, in order to obtain the largest possible value of the coupling, the fields should be untwisted or twisted associated to the same fixed point. In both cases $\partial_{i} \lambda_{\alpha \beta \gamma} \rightarrow 0$, and we will only consider this possibility here.

Using the above information, one can analyse the structure of soft parameters available in Abelian orbifolds. In the dilaton-dominated SUSY-breaking case $(\cos \theta=$ $0)$ the soft parameters are universal, and fulfil $[50,51]$

$$
m=m_{3 / 2}, M= \pm \sqrt{3} m, A=-M
$$

where the positive (negative) $\operatorname{sign}$ for $M$ corresponds to $\theta=\pi / 2(\theta=3 \pi / 2)$. Of course, these are a subset of the parameter space of mSUGRA, and as a consequence one should expect small dark matter detection cross sections, as discussed in the previous Section.

However, in general, the soft terms (scalar masses and trilinear parameters) given in Eq. (3.9) show a lack of universality due to the modular weight dependence. For example, assuming an overall modulus (i.e., $T=T_{i}$ and $\Theta_{i}=1 / \sqrt{3}$ ), one obtains

$$
\begin{aligned}
m_{\alpha}^{2} & =m_{3 / 2}^{2}\left(1+n_{\alpha} \cos ^{2} \theta\right) \\
A_{\alpha \beta \gamma} & =-\sqrt{3} m_{3 / 2} \sin \theta-m_{3 / 2} \cos \theta\left(3+n_{\alpha}+n_{\beta}+n_{\gamma}\right)
\end{aligned}
$$

where we have defined the overall modular weights $n_{\alpha}=\sum_{i} n_{\alpha}^{i}$. In the case of $Z_{n}$ Abelian orbifolds, these can take the values $-1,-2,-3,-4,-5$. Fields belonging to the untwisted sector of the orbifold have $n_{\alpha}=-1$. Fields in the twisted sector but 
without oscillators have usually modular weight -2 , and those with oscillators have $n_{\alpha} \leq-3$. Of course, if all modular weights of the standard model fields are equal, one recovers the universal scenario. For example, taking all $n_{\alpha}=-1$ one has [47] $m=m_{3 / 2} \sin \theta, M=\sqrt{3} m, A=-M$.

Using notation (2.1) and (2.2), the degree of non-universality in the scalar masses is therefore given by

$$
\delta_{\alpha}=n_{\alpha} \cos ^{2} \theta
$$

It is worth noticing here that $\delta_{\alpha} \leq 0$ as a consequence of the negativeness of the modular weights. As we will see, this has important phenomenological implications.

On the other hand, the apparent success of the joining of gauge coupling constants at, approximately, $2 \times 10^{16} \mathrm{GeV}$ in the MSSM is not automatic in the heterotic superstring, where the natural unification scale is $M_{G U T} \simeq g_{G U T} \times 5.27 \times 10^{17} \mathrm{GeV}$, where $g_{G U T}$ is the unified gauge coupling. Therefore unification takes place at energies around a factor 10 smaller than expected in the heterotic superstring. This problem might be solved with the presence of large string threshold corrections which explain the mismatch between both scales $[52,53]$. In a sense, what would happen is that the gauge coupling constants cross at the MSSM unification scale and diverge towards different values at the heterotic string unification scale. These different values appear due to large one-loop string threshold corrections.

It was found that these corrections can be obtained for restricted values of the modular weights of the fields [53]. In fact, assuming generation independence for the $n_{\alpha}$ as well as $-3 \leq n_{\alpha} \leq-1$, the simplest possibility corresponds to taking the following values for the standard model fields:

$$
\begin{aligned}
& n_{Q_{L}}=n_{d_{R}}=-1, \quad n_{u_{R}}=-2, \quad n_{L_{L}}=n_{e_{R}}=-3, \\
& n_{H_{u}}+n_{H_{d}}=-5,-4,
\end{aligned}
$$

where, e.g., $u_{R}$ denotes the three family squarks $\tilde{u}_{R}, \tilde{c}_{R}, \tilde{t}_{R}$. The above values together with $\operatorname{Re} T \simeq 16$ lead to good agreement for $\sin ^{2} \theta_{W}$ and $\alpha_{3}$ [53]. The associated soft sfermion masses are given by [47]:

$$
\begin{aligned}
m_{Q_{L}}^{2}, m_{d_{R}}^{2} & =m_{3 / 2}^{2}\left(1-\cos ^{2} \theta\right), \\
m_{u_{R}}^{2} & =m_{3 / 2}^{2}\left(1-2 \cos ^{2} \theta\right), \\
m_{L_{L}}^{2}, m_{e_{R}}^{2} & =m_{3 / 2}^{2}\left(1-3 \cos ^{2} \theta\right),
\end{aligned}
$$

whereas for the soft Higgs masses, choosing $n_{H_{u}}=-1, n_{H_{d}}=-3$, one obtains:

$$
m_{H_{u}}^{2}=m_{3 / 2}^{2}\left(1-\cos ^{2} \theta\right),
$$




\begin{tabular}{|c|ccccccc|}
\hline & $n_{Q_{L}}$ & $n_{u_{R 1,2,3}}$ & $n_{d_{R}}$ & $n_{L_{L}}$ & $n_{e_{R 1,2,3}}$ & $n_{H_{d}}$ & $n_{H_{u}}$ \\
\hline A) & -1 & -2 & -1 & -3 & -3 & -3 & -1 \\
\hline B) & -1 & -2 & -1 & -3 & -3 & -3 & -2 \\
\hline C) & -1 & -2 & -2 & -1 & -1 & -2 & -1 \\
\hline D) & -2 & -1 & -1 & -2 & -1 & -2 & -2 \\
\hline E) & -1 & $-1,-3,-3$ & -1 & -3 & $-1,-3,-3$ & -2 & -3 \\
\hline
\end{tabular}

Table 1: Modular weights for the scalar fields of heterotic orbifold scenarios with an overall modulus that can reproduce gauge unification $[50,53]$. Note that cases B) and C) present extra massless chiral fields.

$$
m_{H_{d}}^{2}=m_{3 / 2}^{2}\left(1-3 \cos ^{2} \theta\right) .
$$

For convenience, this set of modular weights is summarised in Table 1 and labelled as case A).

For example, with $\cos ^{2} \theta=1 / 3$, using notation (2.1) and (2.2), the non-universalities in the Higgs and sfermion sectors correspond to $\delta_{H_{u}}=-1 / 3, \delta_{H_{d}}=-1, \delta_{Q_{L}}=\delta_{d_{R}}=$ $-1 / 3, \delta_{u_{R}}=-2 / 3$, and $\delta_{L_{L}}=\delta_{e_{R}}=-1$.

Concerning the soft gaugino masses, they are given by:

$$
\begin{aligned}
& M_{3} \simeq 1.0 \sqrt{3} m_{3 / 2} \sin \theta \\
& M_{2} \simeq 1.06 \sqrt{3} m_{3 / 2} \sin \theta \\
& M_{1} \simeq 1.18 \sqrt{3} m_{3 / 2} \sin \theta
\end{aligned}
$$

The small departure from universality is due to the effect of the string threshold corrections on the gauge kinetic function [47].

Finally, for the above modular weights, and using (3.12), the expressions for the trilinear parameters read

$$
\begin{aligned}
& A_{\tau}=-m_{3 / 2}(\sqrt{3} \sin \theta-6 \cos \theta), \\
& A_{b}=-m_{3 / 2}(\sqrt{3} \sin \theta-2 \cos \theta), \\
& A_{t}=-m_{3 / 2}(\sqrt{3} \sin \theta-\cos \theta) .
\end{aligned}
$$

The $A$-term which is relevant to radiative symmetry breaking is the one associated to the top-quark Yukawa coupling $A_{t}$.

These soft terms serve as an explicit model for the study of the neutralino detection cross section. Since they are completely determined in terms of just the gravitino mass 


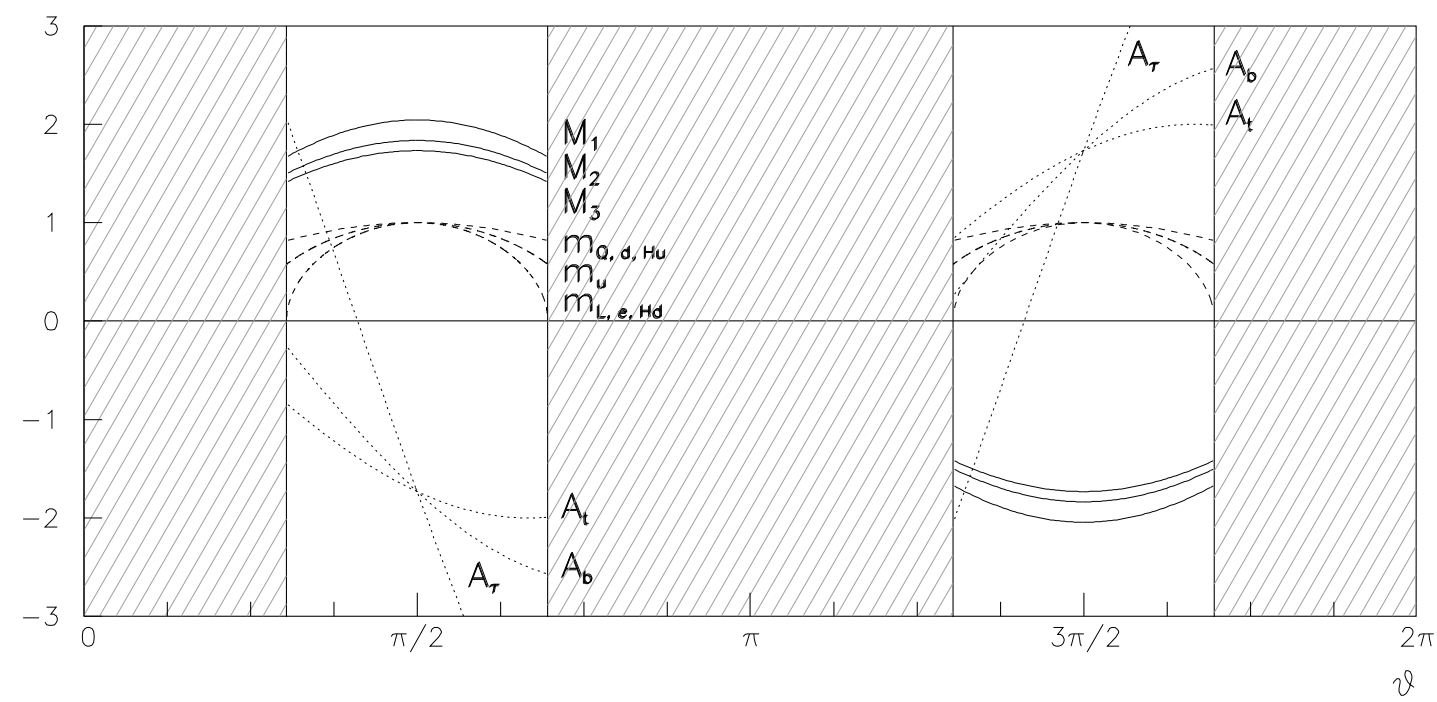

Figure 1: Soft terms at the string scale in units of $m_{3 / 2}$ as a function of the Goldstino angle, $\theta$. Solid lines represent, from top to bottom, the bino, wino and gluino mass parameters. The various scalar masses are depicted by means of dashed lines. Finally, dotted lines correspond to the trilinear terms. The oblique ruled areas are excluded due to the occurrence of negative mass-squared parameters.

and the Goldstino angle, we are left with three free parameters, namely $m_{3 / 2}, \theta$, and $\tan \beta$, plus the sign of $\mu$. Note, however, that the absence of negative mass-squared of the sleptons at the GUT scale implies the constraint $\cos ^{2} \theta \leq \frac{1}{3}$. Besides, the shift $\theta \rightarrow \theta+\pi$ implies in the above equations $m_{\alpha} \rightarrow m_{\alpha}, M_{a} \rightarrow-M_{a}$ and $A_{t} \rightarrow-A_{t}$. This fact makes it unnecessary to consider both signs of the $\mu$ parameter. The reason is that the RGEs are symmetric under the change $\mu, M, A \rightarrow-\mu,-M,-A$. Consequently, in the remainder of this paper we will assume $\mu>0$. Notice in this sense that we will always have $\mu M_{i}>0$ for $\theta<\pi$ whereas $\mu M_{i}<0$ for $\theta>\pi$. This will have important implications, as we will soon see, on the effect of the experimental constraints on the rare decays $b \rightarrow s \gamma$ and $B_{s}^{0} \rightarrow \mu^{+} \mu^{-}$, and on the SUSY contribution to the muon anomalous magnetic moment, $a_{\mu}^{\mathrm{SUSY}}$.

The resulting structure of the soft parameters for case A), given at the GUT scale, is represented in Fig. 1 as a function of the Goldstino angle in units of the gravitino mass. Two generic features of this kind of orbifold constructions are evidenced by the plot, namely, the fact that scalar masses are always smaller than gaugino masses, and the presence of regions which are excluded because some scalar masses-squared become negative. In the present example, as already mentioned, the strongest bound is set by 

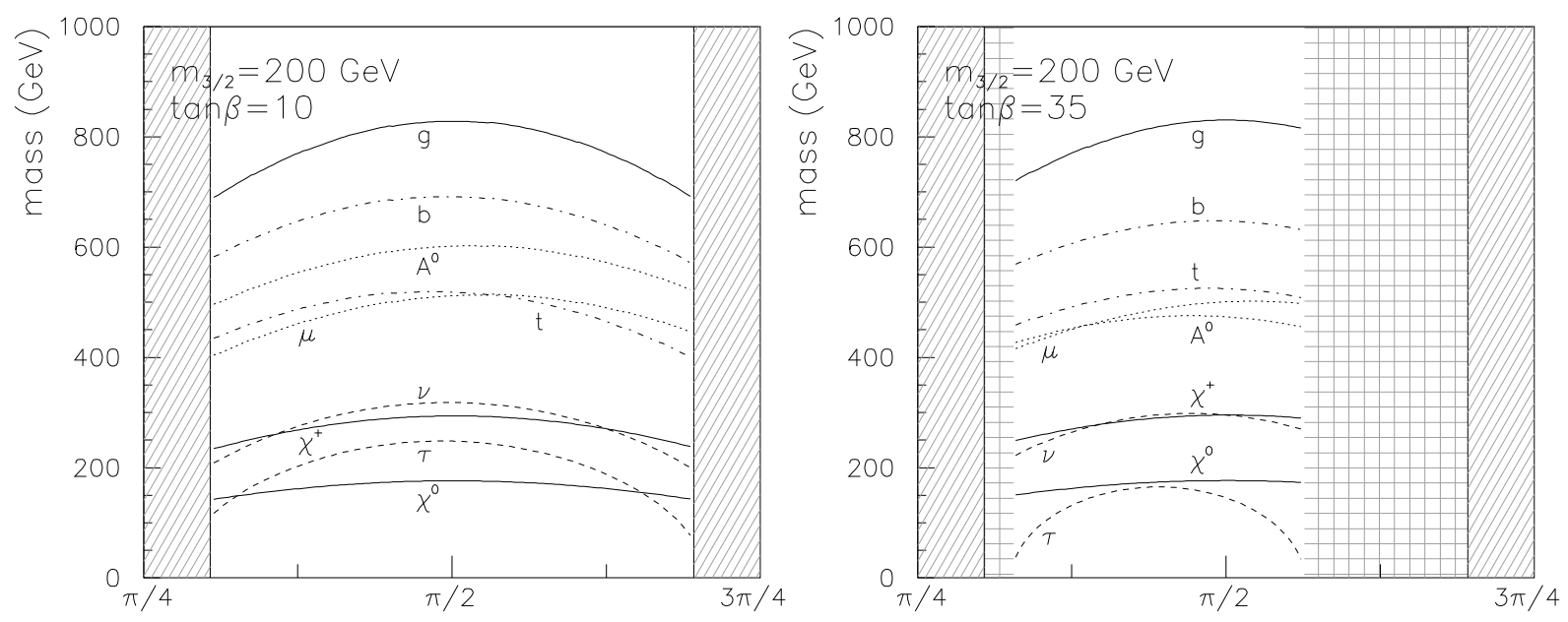

Figure 2: Supersymmetric spectrum at low-energy as a function of the Goldstino angle, $\theta$, for $m_{3 / 2}=200 \mathrm{GeV}$ and $\tan \beta=10$ and 35 . Only the region around $\theta=\pi / 2$ is represented. From bottom to top, the solid lines correspond to the lightest neutralino, lightest chargino and gluino masses. The dashed lines represent the lightest stau and lightest sneutrino masses. The lightest stop and sbottom masses are plotted by means of dot-dashed lines. Finally, the dotted lines show the mass of the CP-odd Higgs and the resulting $\mu$ parameter. The oblique ruled areas are excluded due to the occurrence of negative mass-squared parameters at the GUT scale, whereas the gridded regions correspond to those where tachyons appear after solving the RGEs.

slepton masses, for which (3.15) implies $\cos ^{2} \theta \leq 1 / 3$. The ruled areas correspond to those where this bound is not fulfilled. This reduces the allowed parameter space to two strips in $\theta$, around the dilaton-dominated case, $\theta=\pi / 2,3 \pi / 2$.

With this information, the RGEs are numerically solved and the low-energy supersymmetric spectrum is calculated. Fig. 2 shows the resulting particle spectrum as a function of the Goldstino angle for $m_{3 / 2}=200 \mathrm{GeV}$ and $\tan \beta=10$ and 35. As we can see, although slepton masses-squared are positive at the GUT scale for $\cos ^{2} \theta \leq 1 / 3$, the RGEs can still drive them negative, or lead to tachyonic mass eigenstates. This is typically the case of the lightest stau, $\tilde{\tau}_{1}$, and lightest sneutrino, $\tilde{\nu}_{1}$ (the latter only for low values of the gravitino mass), due to their small mass parameters (3.15). This is more likely to happen for large $\tan \beta$, since the lepton Yukawas (which are proportional to $1 / \cos \beta$ ) increase and induce a larger negative contribution to the slepton RGEs. In such a case, the lightest stau can be the lightest SUSY particle (LSP) in larger regions 
of the parameter space, thus potentially reducing the allowed areas for neutralino dark matter, as we see in the example with $\tan \beta=35$. The supersymmetric spectrum also displays a heavy squark sector, due to the gluino contribution on the running of their mass parameters. Similarly, the heavy Higgs masses (represented here only with the pseudoscalar, $A^{0}$ ) are also sizable. For reference, the value of the $\mu$ term is also displayed and found to be large.

Notice at this point that there are regions of the parameter space where the lightest neutralino is the LSP and the stau, being the next-to-lightest SUSY particle (NLSP), has a very similar mass. As we will soon see, this allows reproducing the correct dark matter relic density by means of a coannihilation effect. On the other hand, one can readily see that in these examples $m_{A^{0}}>2 m_{\tilde{\chi}_{1}^{0}}$ and therefore there is no enhancement in the annihilation of neutralinos mediated by the CP-odd Higgs.

Finally, it is worth emphasizing that in these scenarios the gravitino is never the LSP. Despite the bino mass being larger than $m_{3 / 2}$ at the string scale, its RGE always leads to $M_{1}<m_{3 / 2}$ at the electroweak scale (even in the dilaton-dominated limit for which $M_{1}$ is at its maximum) so that, at least, the neutralino mass is always lighter than $m_{3 / 2}$.

Having extracted the supersymmetric spectrum, we are ready to determine the implications for low-energy observables and study how the associated bounds further restrict the allowed parameter space. In our analysis the most recent experimental and astrophysical constraints have been included. In particular, the lower bounds on the masses of the supersymmetric particles and on the lightest Higgs have been implemented, as well as the experimental bound on the branching ratio of the $b \rightarrow$ $s \gamma$ process, $2.85 \times 10^{-4} \leq \mathrm{B}(b \rightarrow s \gamma) \leq 4.25 \times 10^{-4}$. The latter has been calculated taking into account the most recent experimental world average for the branching ratio reported by the Heavy Flavour Averaging Group [54-56], as well as the new reevaluation of the SM value [57], with errors combined in quadrature. We also take into account the improved experimental constraint on the $B_{s}^{0} \rightarrow \mu^{+} \mu^{-}$branching ratio, $\mathrm{B}\left(B_{s}^{0} \rightarrow \mu^{+} \mu^{-}\right)<1.5 \times 10^{-7}$, obtained from a combination of the results of CDF and D0, [58-60]. The evaluation of the neutralino relic density is carried out with the program micrOMEGAs [61], and, due to its relevance, the effect of the WMAP constraint will be shown explicitly. Finally, dangerous charge and colour breaking minima of the Higgs potential will be avoided by excluding unbounded from below directions.

Concerning $a_{\mu}^{\mathrm{SUSY}}$, we have taken into account the experimental result for the muon anomalous magnetic moment [62], as well as the most recent theoretical evaluations of 
the Standard Model contributions [63-65]. It is found that when $e^{+} e^{-}$data are used the experimental excess in $a_{\mu} \equiv\left(g_{\mu}-2\right) / 2$ would constrain a possible supersymmetric contribution to be $a_{\mu}^{\text {SUSY }}=(27.6 \pm 8) \times 10^{-10}$, where theoretical and experimental errors have been combined in quadrature. However, when tau data are used, a smaller discrepancy with the experimental measurement is found. Due to this reason, in our analysis we will not impose this constraint, but only indicate the regions compatible with it at the $2 \sigma$ level, this is, $11.6 \times 10^{-10} \leq a_{\mu}^{\mathrm{SUSY}} \leq 43.6 \times 10^{-10}$.

For a better understanding of all these constraints, we have represented in Fig. 3 their effect on the $\left(m_{3 / 2}, \theta\right)$ plane for $\tan \beta=10$. For comparison, the cases with $\tan \beta=20,35$ are also shown in Fig. 4 .

The first thing to notice is that extensive regions are excluded due to the occurrence of tachyonic masses for sleptons. As already discussed, the area excluded for this reason becomes larger when $\tan \beta$ increases, an effect which is clearly displayed in Figs. 3 and 4. This implies an increase in the lower bound of the gravitino mass. Whereas for $\tan \beta=10,20$ the smallest allowed value is $m_{3 / 2} \approx 35 \mathrm{GeV}$, in the case with $\tan \beta=35$ one needs $m_{3 / 2} \gtrsim 90 \mathrm{GeV}$.

The above mentioned smallness of the slepton mass parameters, together with the fact that gaugino masses are always larger than scalar masses $\left(M_{a}>m_{\alpha}\right)$, also imply that the areas in the parameter space where the lightest neutralino is the LSP are not very extensive. These regions occur for small values of $\cos \theta$ (they are centered around $\theta \approx \pi / 2,3 \pi / 2)$, since the ratio $|M| / m_{L_{L}, e_{R}}$ increases with ${ }^{4} \cos \theta$. Note that such values of the Goldstino angle mean that the breaking of SUSY is mainly due to the dilaton auxiliary term. Once more, the allowed areas shrink for large values of $\tan \beta$ and eventually disappear for $\tan \beta \approx 45$. In the rest of the parameter space the role of the LSP is mainly played by the lightest stau. Although, as already mentioned, for small values of $\tan \beta$ the sneutrino can also be the LSP in a very narrow band for small gravitino masses, this area is always excluded by experimental bounds.

The relevance of the experimental constraints is also evidenced by Figs. 3 and 4. Reproducing the experimental result of the branching ratio of $b \rightarrow s \gamma$ is much easier in the region around $\theta=\pi / 2$, since it has $\mu M>0$. On the contrary, it poses a stringent lower bound on the value of $m_{3 / 2}$ for the region around $\theta=3 \pi / 2$, for which $\mu M<0$. As expected, the area excluded for this reason also increases for larger values of $\tan \beta$. Thus, whereas this constraint implies $m_{3 / 2} \gtrsim 150 \mathrm{GeV}$ for $\tan \beta=10$ in the

\footnotetext{
${ }^{4}$ The lack of a complete mirror symmetry at $\theta=\pi / 2$ and $3 \pi / 2$ is due to the trilinear terms $(3.12)$ being a combination of $\sin \theta$ and $\cos \theta$.
} 


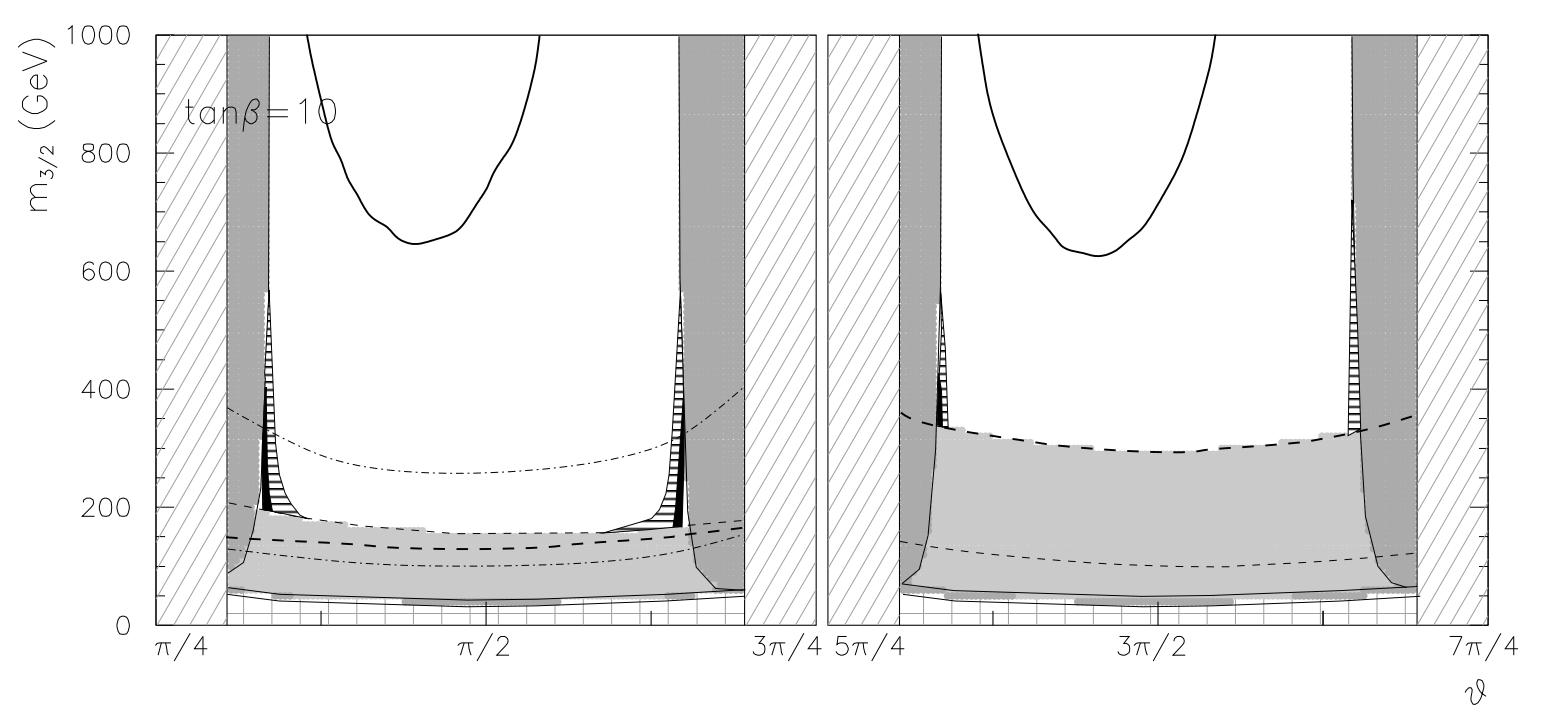

Figure 3: Effect of the different experimental constraints on the parameter space $\left(m_{3 / 2}, \theta\right)$ for $\tan \beta=10$. The oblique ruled areas are excluded due to the occurrence of tachyons at the GUT scale, whereas the gridded regions correspond to those where tachyons appear after solving the RGEs. Only the two areas centred around $\theta \approx \pi / 2,3 \pi / 2$ are free from tachyons in the slepton sector. Dark grey areas represent those where the lightest neutralino is not the LSP. Among these regions, the narrow vertical areas contained within solid lines are those where the stau is the LSP, whereas in the thin horizontal region at very small gravitino masses, also bounded by solid lines, the LSP is the lightest sneutrino. Light grey areas stand for those not fulfilling one or more experimental bounds. In particular, the region below the thin dashed line is excluded by the lower bound on the Higgs mass. The area below the thick dashed line is excluded by $b \rightarrow s \gamma$. The regions excluded by the experimental constraints on the masses of the chargino and stau are always contained within those ruled out by other reasons and are therefore not shown. The region bounded by thin dot-dashed lines is favoured by $a_{\mu}^{\mathrm{SUSY}}$ (notice that the whole allowed area around $\theta \approx 3 \pi / 2$ always has $a_{\mu}^{\mathrm{SUSY}}<0$ and is therefore disfavoured), although this constraint has not been explicitly applied. The remaining white area is favoured by all the experimental constraints. Within it the ruled region fulfils in addition $0.1 \leq \Omega_{\tilde{\chi}_{1}^{0}} h^{2} \leq 0.3$, and the black area on top of this indicates the WMAP range $0.094<\Omega_{\tilde{\chi}_{1}^{0}} h^{2}<0.112$. Finally, the UFB constraints are only fulfilled in the area above the thick solid line.

area around $\theta=\pi / 2, m_{3 / 2} \gtrsim 250(300) \mathrm{GeV}$ is necessary for $\tan \beta=20$ (35).

Having $\mu M<0$, the whole region around $\theta=3 \pi / 2$ also fails to fulfil the experimental constraint on $a_{\mu}^{\mathrm{SUSY}}$, and is therefore further disfavoured. 


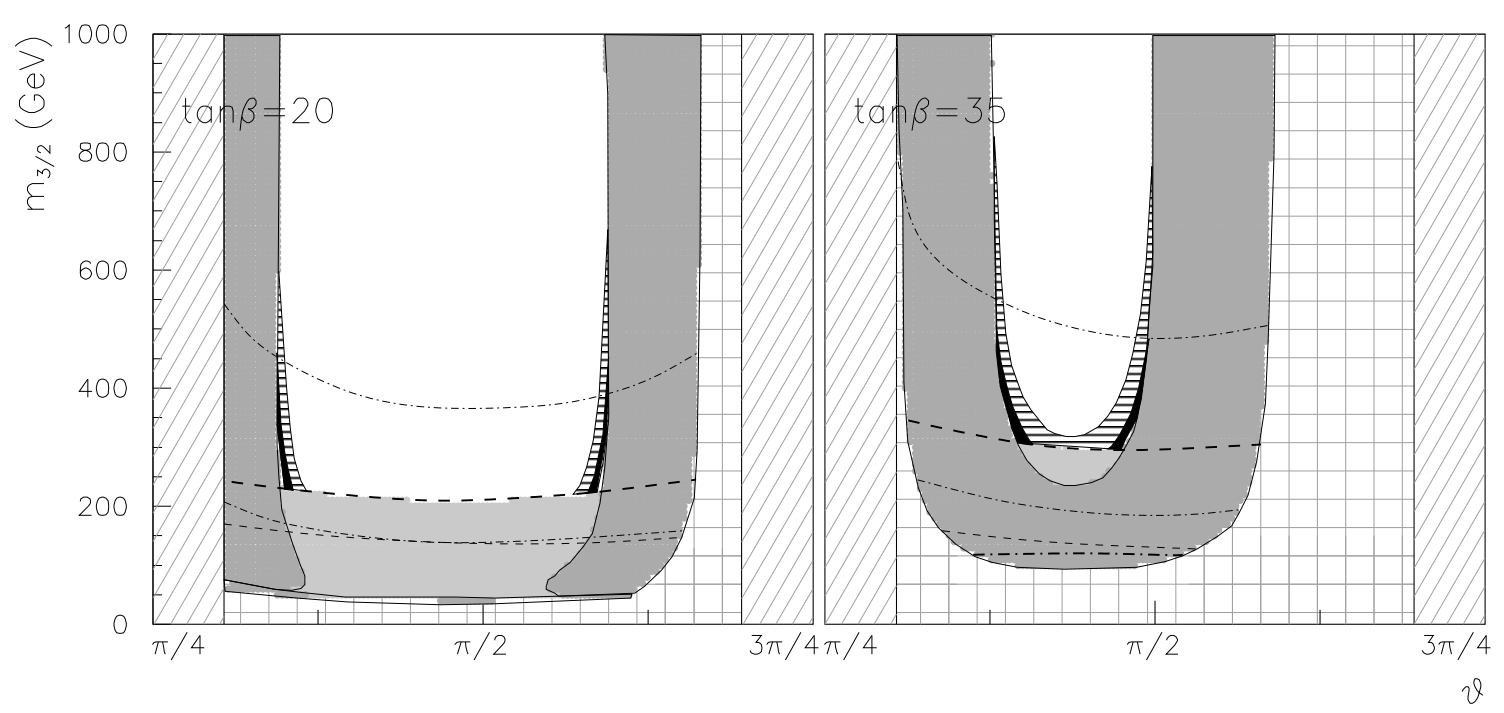

Figure 4: The same as Fig. 3 but for $\tan \beta=20$ and 35. Notice that the UFB constraints are violated in the whole represented parameter space and therefore no thick solid line is shown. In the plot for $\tan \beta=35$, the area below the thick dot-dashed line on the region is excluded due to the constraint on the $B_{s}^{0} \rightarrow \mu^{+} \mu^{-}$branching ratio

The bound on the lightest Higgs mass also rules out some regions for small gravitino masses. This is only relevant for small values of $\tan \beta$ and in the region around $\theta=\pi / 2$. Already for $\tan \beta \gtrsim 15$ this bound becomes less important than the $b \rightarrow s \gamma$ or $a_{\mu}^{\mathrm{SUSY}}$ constraints. The areas not fulfilling the experimental constraints on sparticle masses are always contained within those already excluded by other bounds and are therefore not shown explicitly.

The allowed parameter space is further reduced when the constraint on the relic density is imposed. The WMAP result is only reproduced along the narrow regions close to the area where the stau becomes the LSP. This is due to the well known coannihilation effect that takes place when the neutralino mass is close to the stau mass. The equivalent of the "bulk region" in the mSUGRA parameter space is here excluded by the experimental constraints. Finally, no regions are found where $2 m_{\tilde{\chi}_{1}^{0}} \approx m_{A}$, and consequently resonant annihilation of neutralinos does not play any role in this case.

Having shown that there are regions with viable neutralino dark matter, let us now turn our attention to its possible direct detection. Following the discussion of Section 2, the Higgs modular weights giving rise to the soft masses (3.16), could induce an increase of the neutralino detection cross section with respect to the universal case. In 

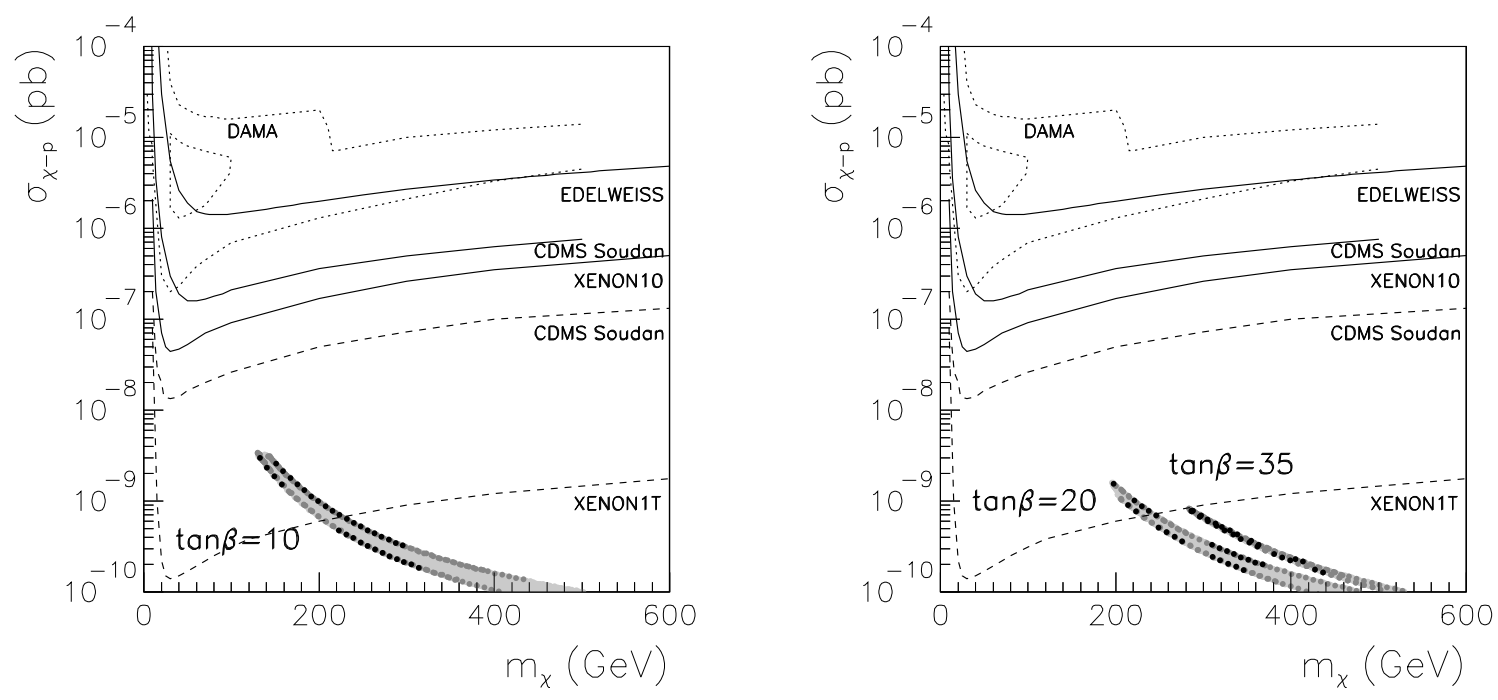

Figure 5: Scatter plot of the scalar neutralino-proton cross section, $\sigma_{\tilde{\chi}_{1}^{0}-p}$, as a function of the neutralino mass, $m_{\tilde{\chi}_{1}^{0}}$, for $\tan \beta=10,20$ and 35 . The light grey dots represent points fulfilling all the experimental constraints. The dark grey dots represent points which satisfy in addition $0.1 \leq \Omega_{\tilde{\chi}_{1}^{0}} h^{2} \leq 0.3$ and the black dots on top of these indicate those in agreement with the WMAP range $0.095<\Omega_{\tilde{\chi}_{1}^{0}} h^{2}<0.112$. The sensitivities of present and projected experiments are also depicted with solid and dashed lines, respectively, in the case of an isothermal spherical halo model. The large (small) area bounded by dotted lines is allowed by the DAMA experiment when astrophysical uncertainties to this simple model are (are not) taken into account.

order to investigate this possibility, the theoretical predictions for the spin-independent part of the neutralino-nucleon cross section have been calculated in the accepted regions of the parameter space. They are represented versus the neutralino mass in Fig. 5 for $\tan \beta=10,20$ and 35, where the sensitivities of present and projected dark matter experiments are also shown. These results resemble those of mSUGRA, as no high values are obtained. As in mSUGRA, in this scenario the $\mu$ parameter and the heavy Higgs masses are sizable (see Fig. 2), thus implying bino-like neutralinos and a suppressed contribution to $\sigma_{\tilde{\chi}_{1}^{0}-p}$ from Higgs-exchanging processes. This is illustrated in Fig. 6, where the resulting values of the $\mu$ parameter are plotted as a function of the pseudoscalar Higgs mass. After analysing the range $\tan \beta=10$ to 50 we found that $\sigma_{\tilde{\chi}_{1}^{0}-p} \lesssim 5 \times 10^{-9} \mathrm{pb}$, the maximum values corresponding to $\tan \beta \approx 15$. These results are therefore beyond the present sensitivities of dark matter detectors and would only be partly within the reach of the projected 1 tonne detectors. 

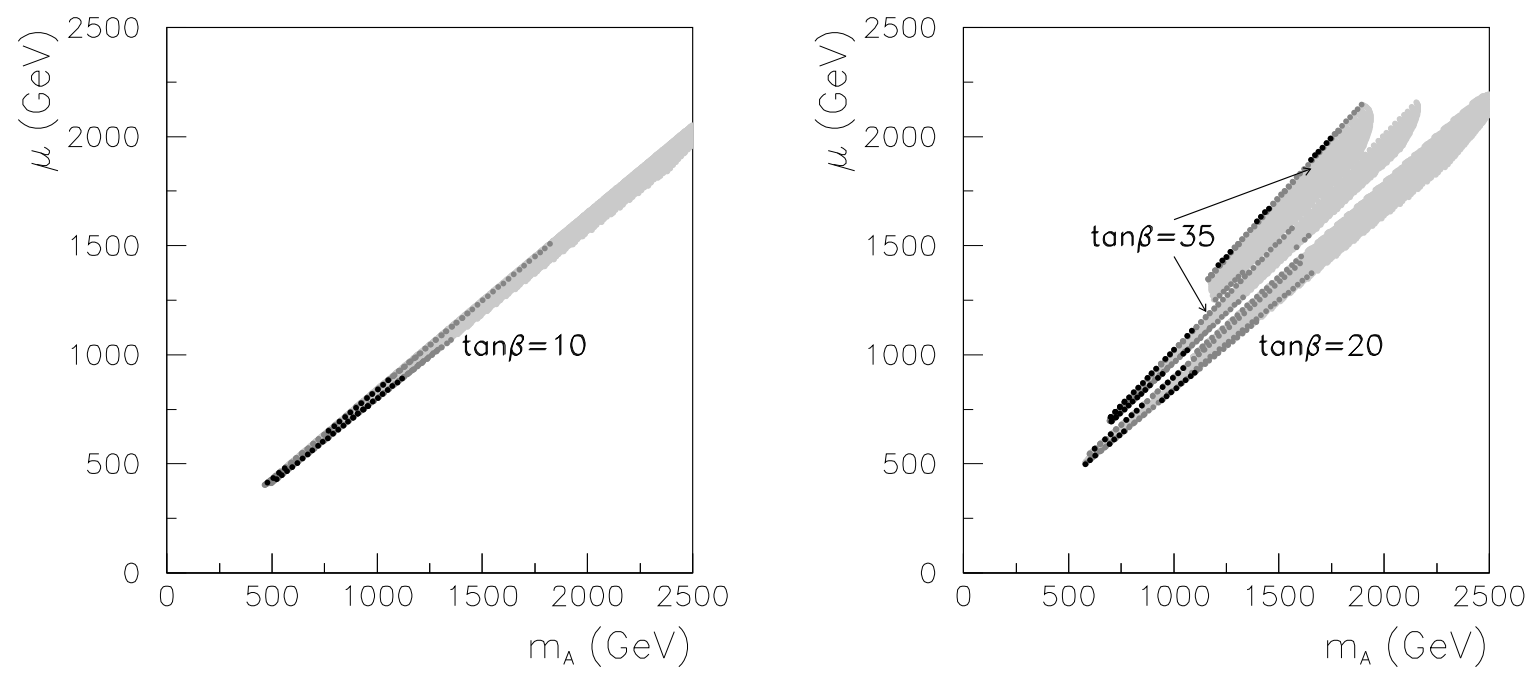

Figure 6: Scatter plot of the resulting $\mu$ parameter as a function of the pseudoscalar Higgs mass, $m_{A}$, for $\tan \beta=10,20$ and 35 . The colour convention is as in Fig. 5.

So far we have not commented on the bounds imposed by the UFB-3 constraint to avoid dangerous charge and colour breaking minima of the Higgs potential. This turns out to play a crucial role in disfavouring this scenario. Indeed, most of the parameter space is excluded on these grounds ${ }^{5}$. Only for small values of $\tan \beta$ and heavy gravitinos do allowed regions appear (see for instance Fig. 3, where $m_{3 / 2} \gtrsim 650 \mathrm{GeV}$ is necessary), but these always correspond to areas where the neutralino relic density is too large and exceeds the WMAP constraint. For $\tan \beta \gtrsim 15$ the UFB-3 constraint already excludes the complete region with $m_{3 / 2}<1000 \mathrm{GeV}$. Once more, the reason for this is the low value of the slepton masses, and more specifically, of the stau mass. Let us recall that the smaller this value, the more negative $V_{U F B-3}$ in (2.4) or (2.5) is, and thus the stronger the UFB-3 bound becomes. Moreover, the fact that in this scenario the value of $m_{H_{u}}^{2}$ is not very large (since $\delta_{H u}$ is negative) also contributes in driving the potential deeper along this direction.

Let us finally remark that other examples with different choices of modular weights for the Higgs parameters satisfying (3.14) have been investigated, such as case B) in Table 1, and lead to qualitatively similar results.

The previous analysis suggests how to modify the model to 'optimise' its behaviour under the UFB-3 constraint [66], increasing also the regions in the parameter space

\footnotetext{
${ }^{5}$ This is consistent with previous analyses of charge and colour breaking minima in different superstring and M-theory scenarios [66].
} 

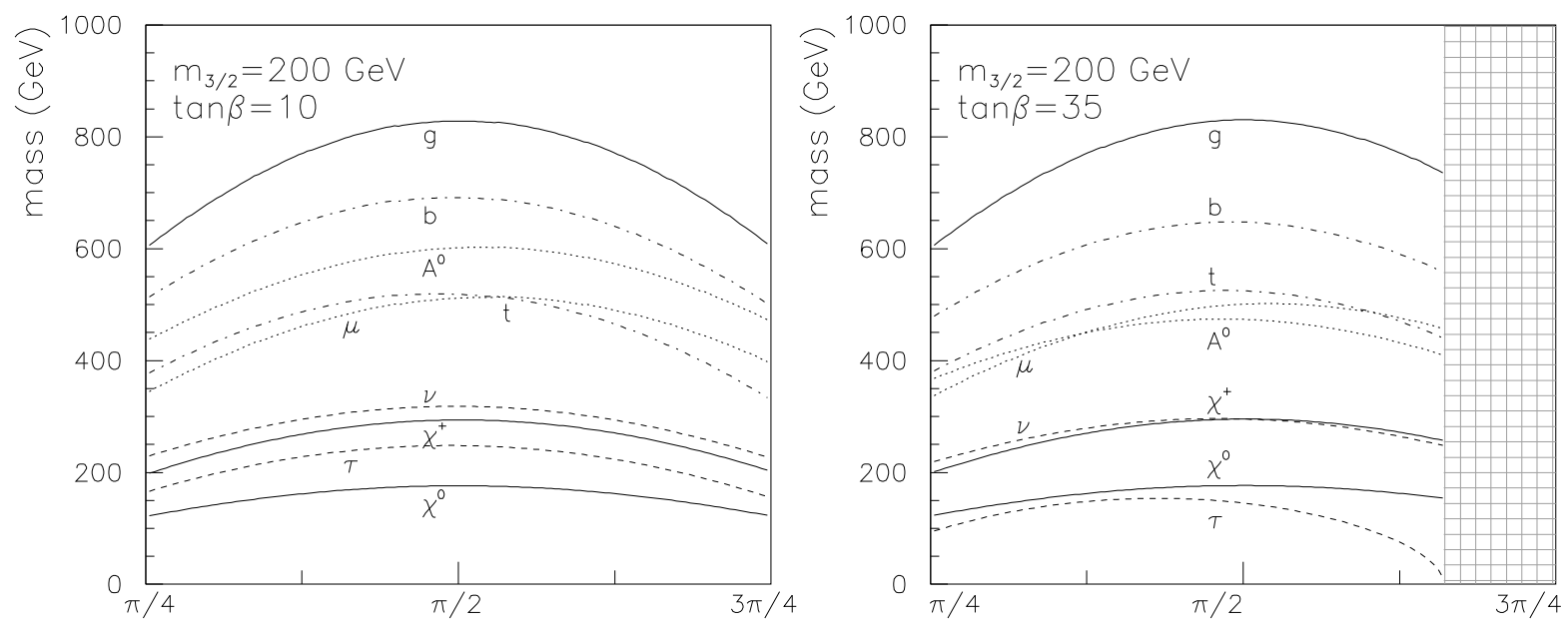

Figure 7: The same as Fig. 2 but for the optimised example where $n_{L_{L}}=n_{e_{R}}=-1$.

where the lightest neutralino is the LSP. The most favorable case would correspond to slepton masses as large as possible, i.e.,

$$
n_{L_{L}}=n_{e_{R}}=-1
$$

For squark and Higgs mass parameters we will continue using the modular weights of case $A)^{6}$. Note that now the bound on $\cos ^{2} \theta$ is less constraining, since we only need to impose $\cos ^{2} \theta \leq \frac{1}{2}$, thus allowing a larger degree of non-universality. For example, with $\cos ^{2} \theta=\frac{1}{3}$, we get $\delta_{H_{u}}=-1 / 3$ and $\delta_{H_{d}}=-1$ for the Higgs masses.

The resulting supersymmetric spectrum is shown in Fig. 7 as a function of the Goldstino angle for $m_{3 / 2}=200 \mathrm{GeV}$ with $\tan \beta=10$ and 35 . Notice that now the whole region with $\theta \in[\pi / 4,3 \pi / 4]$ is free from tachyons at the GUT scale. For small $\tan \beta$ the increase in the slepton mass-squared parameters leaves extensive allowed regions where the neutralino can be the LSP. As expected, larger values of $\tan \beta$ lead to a reduction in the stau mass, which now easily becomes the LSP, and gives rise to tachyons in some regions.

These features are evidenced in Fig. 8, where the corresponding $\left(m_{3 / 2}, \theta\right)$ parameter

\footnotetext{
${ }^{6}$ Of course, with such a choice of modular weights we know that the string threshold corrections cannot account for the joining of gauge couplings at the MSSM unification scale. Thus we will be tacitly assuming that there is some other effect (e.g., the existence of further chiral fields in the spectrum below the heterotic string scale $[19,33,69])$ which appropriately produces the correct lowenergy experimental values for gauge couplings.
} 


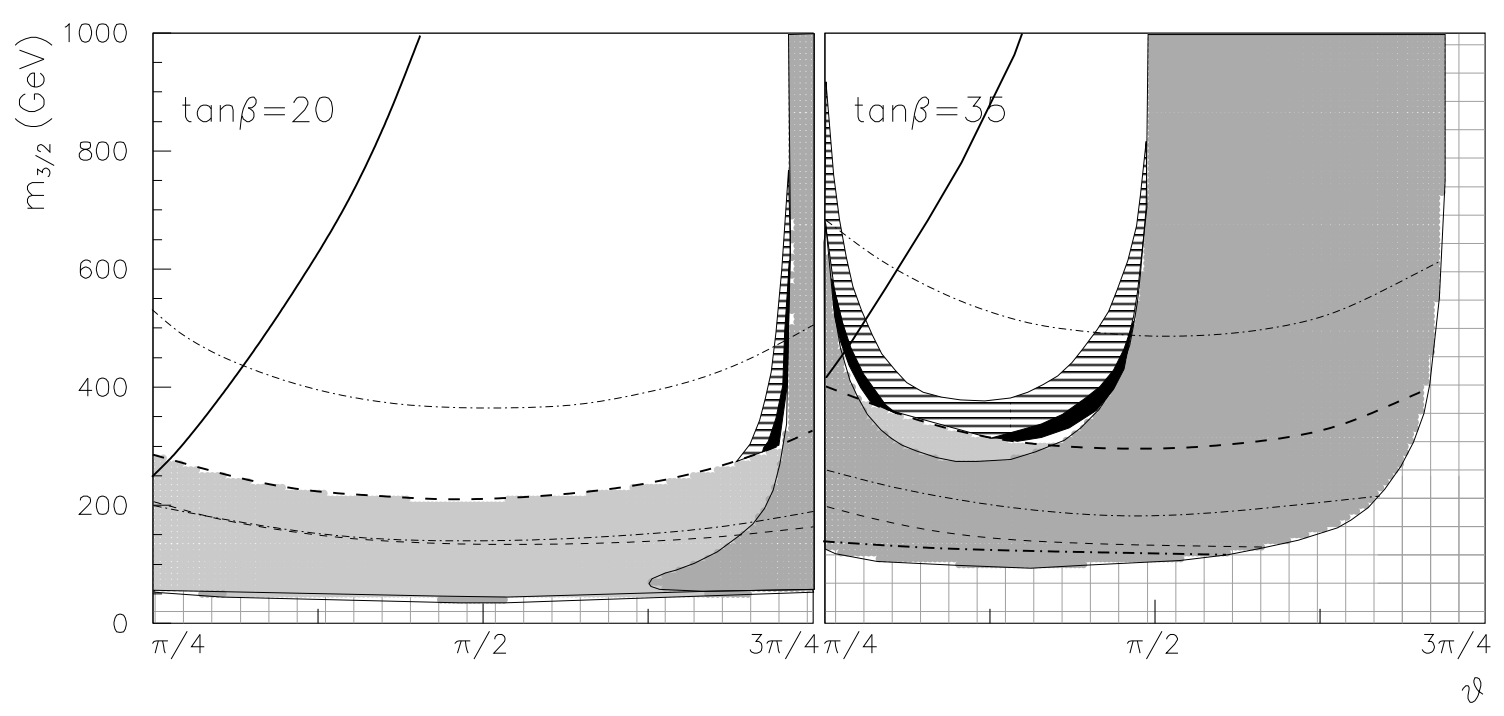

Figure 8: The same as Fig. 3 but for the optimised example where $n_{L_{L}}=n_{e_{R}}=-1$ with $\tan \beta=20,35$.

space is depicted for $\tan \beta=20,35$. Notice that, due to the increase in the slepton mass terms, the stau only becomes the LSP on narrow bands on the right-hand side of the allowed areas for $\tan \beta=20$ (for smaller values of $\tan \beta$ the neutralino is always the LSP). As expected, these areas with stau LSP become more sizable for $\tan \beta=35$ and eventually dominate the whole parameter space for $\tan \beta \gtrsim 45$. Also, for $\tan \beta \lesssim 25$ the sneutrino can also be the LSP on a narrow region for very light gravitinos, although this is always excluded by experimental constraints.

The decrease of the stau mass towards the right-hand side of the allowed areas can be understood by analysing the expressions for the trilinear soft terms. The trilinear terms associated to the top, bottom and tau Yukawa coupling read in this example

$$
\begin{aligned}
A_{t} & =-m_{3 / 2}(\sqrt{3} \sin \theta-\cos \theta), \\
A_{b}=A_{\tau} & =-m_{3 / 2}(\sqrt{3} \sin \theta-2 \cos \theta) .
\end{aligned}
$$

It can be checked that for all of them the ratio $|A / M|$ increases towards the righthand side of both allowed areas. In particular, $\left|A_{\tau, b} / M\right| \approx 0.05$ for $\theta=\pi / 4,5 \pi / 4$ and becomes $\left|A_{\tau, b} / M\right| \approx-1.8$ for $\theta=3 \pi / 4,7 \pi / 4$. The increase in $\left|A_{\tau, b} / M\right|$ leads to a larger negative correction in the RGE for the slepton mass terms, implying lighter staus. Large values of $\tan \beta$ increase the corresponding Yukawas thus further decreasing the stau mass. 

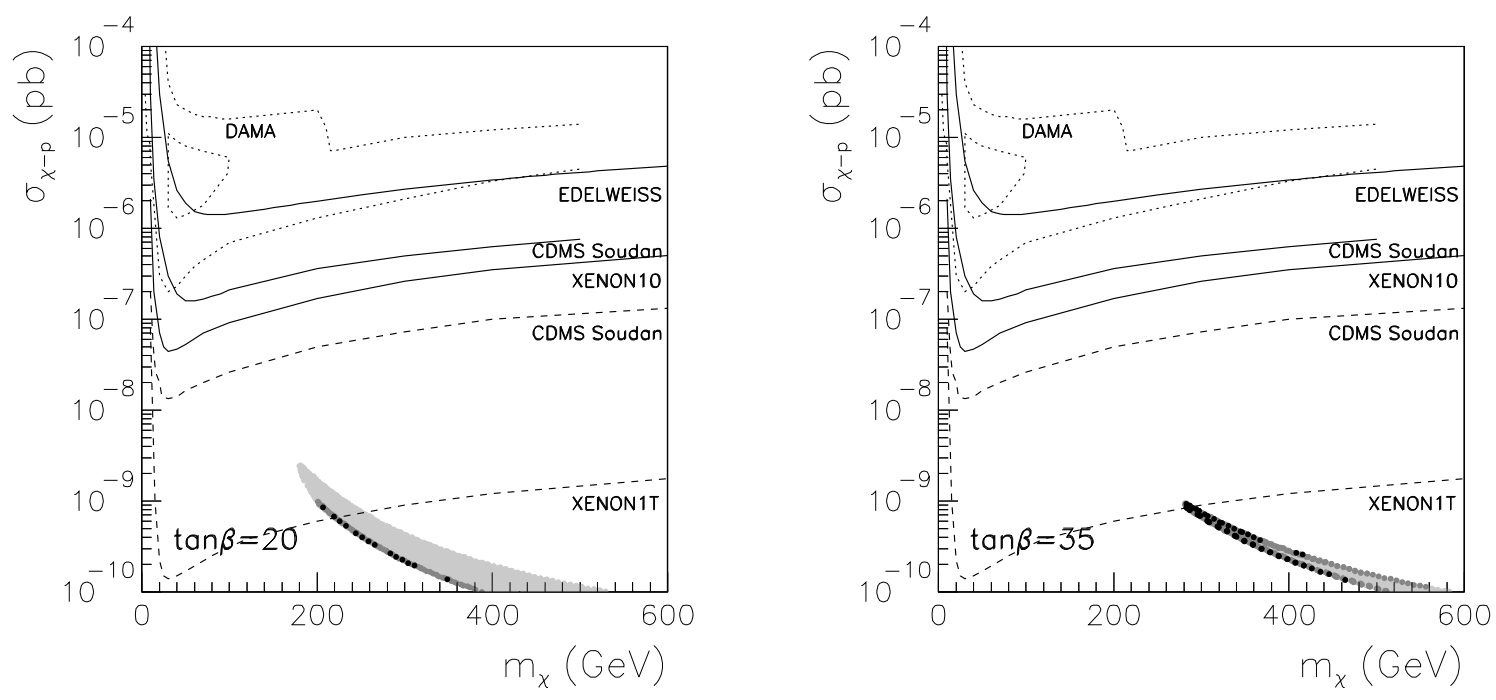

Figure 9: The same as Fig. 5 but for the optimised example where $n_{L_{L}}=n_{e_{R}}=-1$ with $\tan \beta=20,35$.

The variation in the stau mass affects the area excluded by the UFB-3 constraint, which becomes more stringent towards the right-hand side of the allowed regions. On the left, as expected, the effect of the UFB constraints is less severe than in the previous examples and regions with $m_{3 / 2} \gtrsim 250$ (400) $\mathrm{GeV}$ for $\tan \beta=20$ (35) are allowed. Interestingly, for $\tan \beta \gtrsim 30$ part of these areas can also reproduce the correct value for the neutralino relic density.

The corresponding predictions for the neutralino-nucleon cross section are depicted in Fig. 9. Although regions with the correct relic density can appear with $\sigma_{\tilde{\chi}_{1}^{0}-p} \approx 10^{-8}$ $\mathrm{pb}$ for $\tan \beta=20$, the points fulfilling the UFB-3 constraints only correspond to those with $\sigma_{\tilde{\chi}_{1}^{0}-p} \lesssim 10^{-9} \mathrm{pb}$ for $\tan \beta \gtrsim 30$. Once more, as evidenced in Fig. 10, where the resulting $\mu$ parameter is represented versus the CP odd Higgs mass, this is due to the large values of $\mu$ and the heavy Higgs masses.

Notice, finally, that in this example the non-universality of the Higgs masses, given by (3.16), was chosen to be the maximal allowed by the modular weights $\left(n_{H_{u}}=-1\right.$ and $\left.n_{H_{d}}=-3\right)$. Also, the stau mass, for which we have $n_{L, e}=-1$, cannot be further increased and therefore the behaviour under the UFB-3 constraint cannot be improved. Consequently, this optimised scenario represents a good estimate of how large the neutralino detection cross section can be in heterotic orbifolds with overall modulus, where soft masses are given by (3.11). We therefore conclude that in this class of models $\sigma_{\tilde{\chi}_{1}^{0}-p} \lesssim 10^{-8} \mathrm{pb}$. The neutralino in these scenarios would escape detection in 

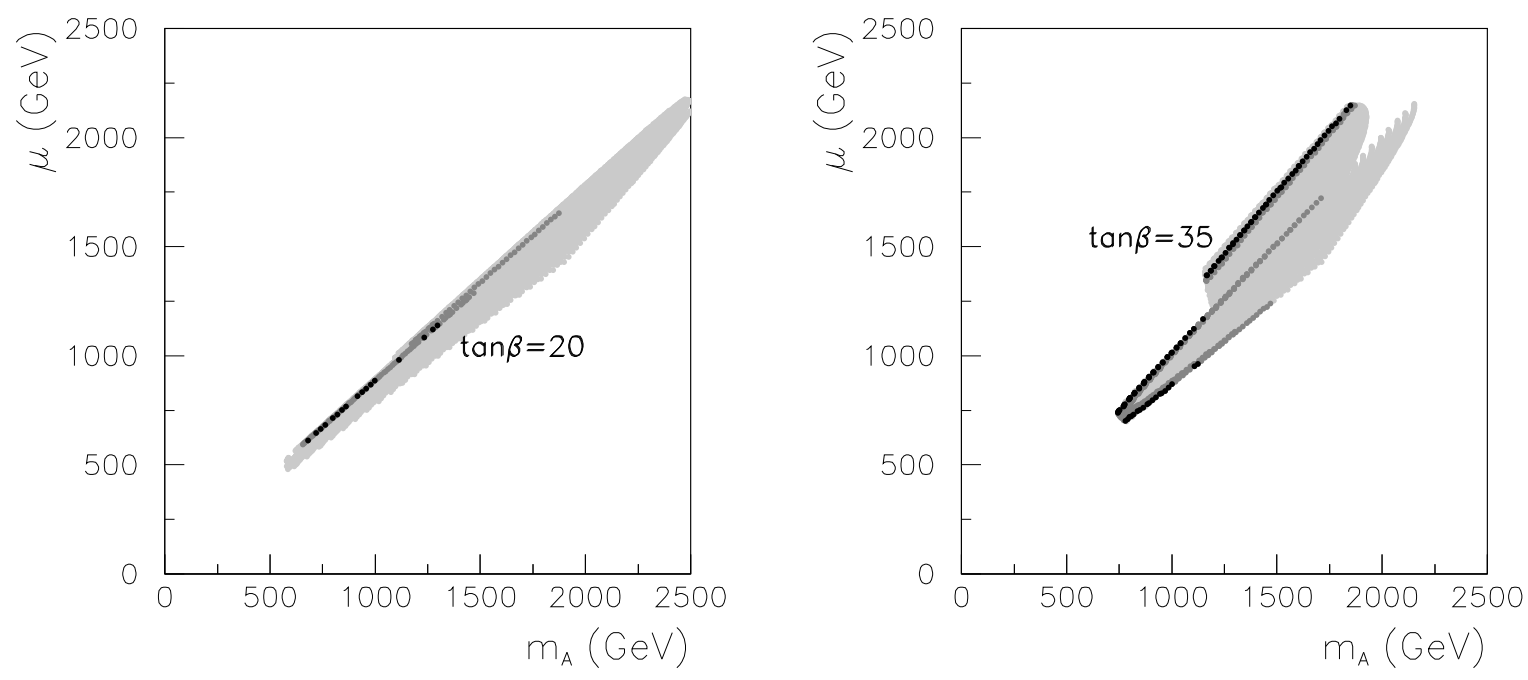

Figure 10: The same as Fig. 6 but for the optimised example where $n_{L_{L}}=n_{e_{R}}=-1$ with $\tan \beta=20,35$.

all present experiments and only 1 tonne detectors would be able to explore some small areas of the allowed parameter space.

For completeness, we have also analysed other three scenarios, described in [50], which also give rise to gauge coupling unification with an overall modulus. Their corresponding modular weights are summarised in Table 1.

For example, in case C) unification is possible with $\operatorname{Re} T=7$, but extra massless chiral fields (one octet, one triplet, and two multiplets transforming like right-handed electrons), with modular weight equal to -1 , are needed. This scenario seems promising, since the modular weights for sleptons are less negative $\left(n_{L, e_{R}}=-1\right)$. In fact, although squarks become tachyonic at the GUT scale for $\cos ^{2} \theta>1 / 2$, sleptons have a positive mass squared in the whole remaining area $\theta \in[\pi / 4,3 \pi / 4],[5 \pi / 4,7 \pi / 4]$. As we have learned from the optimised example, this might be helpful in order to avoid the UFB constraints. The presence of extra matter alters the running of the gauge coupling constants, which are now dictated by the following beta functions, $b_{1}=-13$, $b_{2}=-3$, and $b_{3}=0$. As a consequence, the running of the soft masses is also modified. In particular, all the gaugino masses become smaller at the EW scale, as compared to the usual running within the MSSM. Notice in particular that the gluino mass does not run (at tree level) from the GUT to the EW scales.

The decrease in $M_{1}$ and (especially) $M_{2}$ affect the running of the scalar mass parameters, rendering them smaller at the EW scale. This is enough to offset the increase 

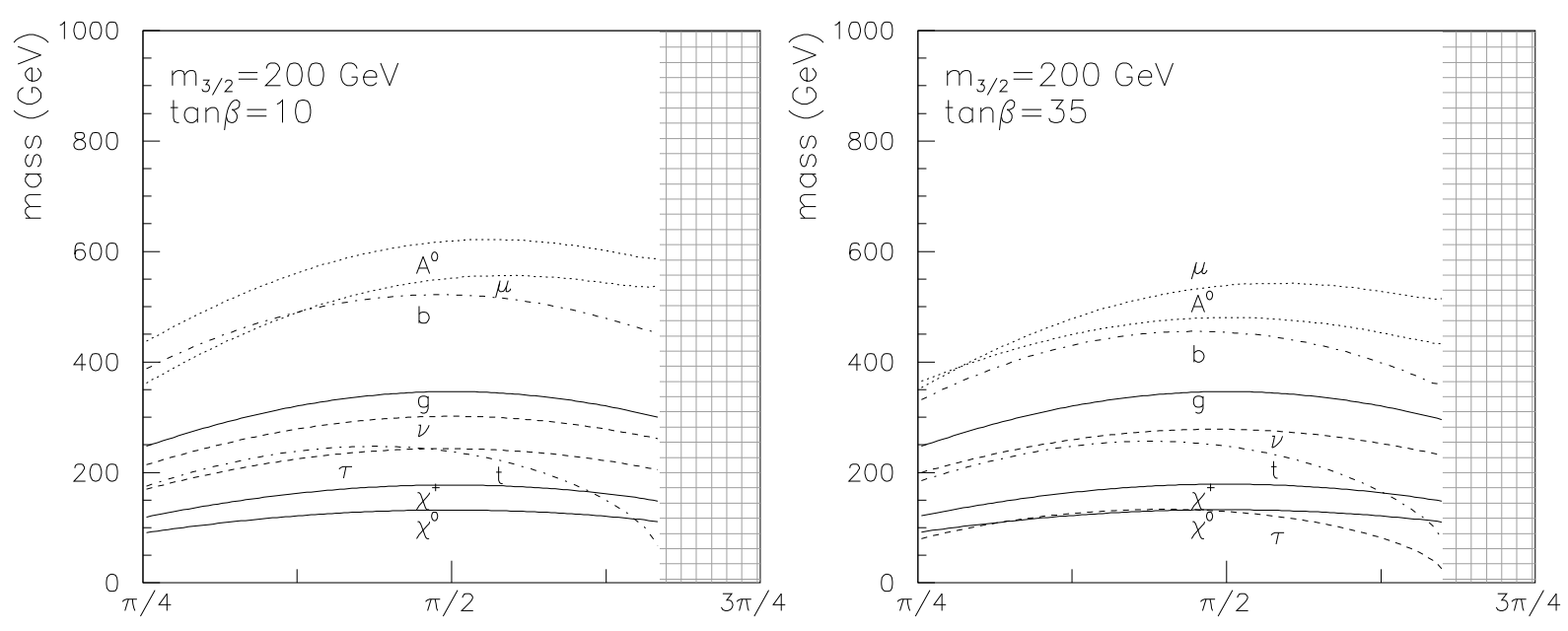

Figure 11: The same as Fig. 2 but for case C) in Table 1.

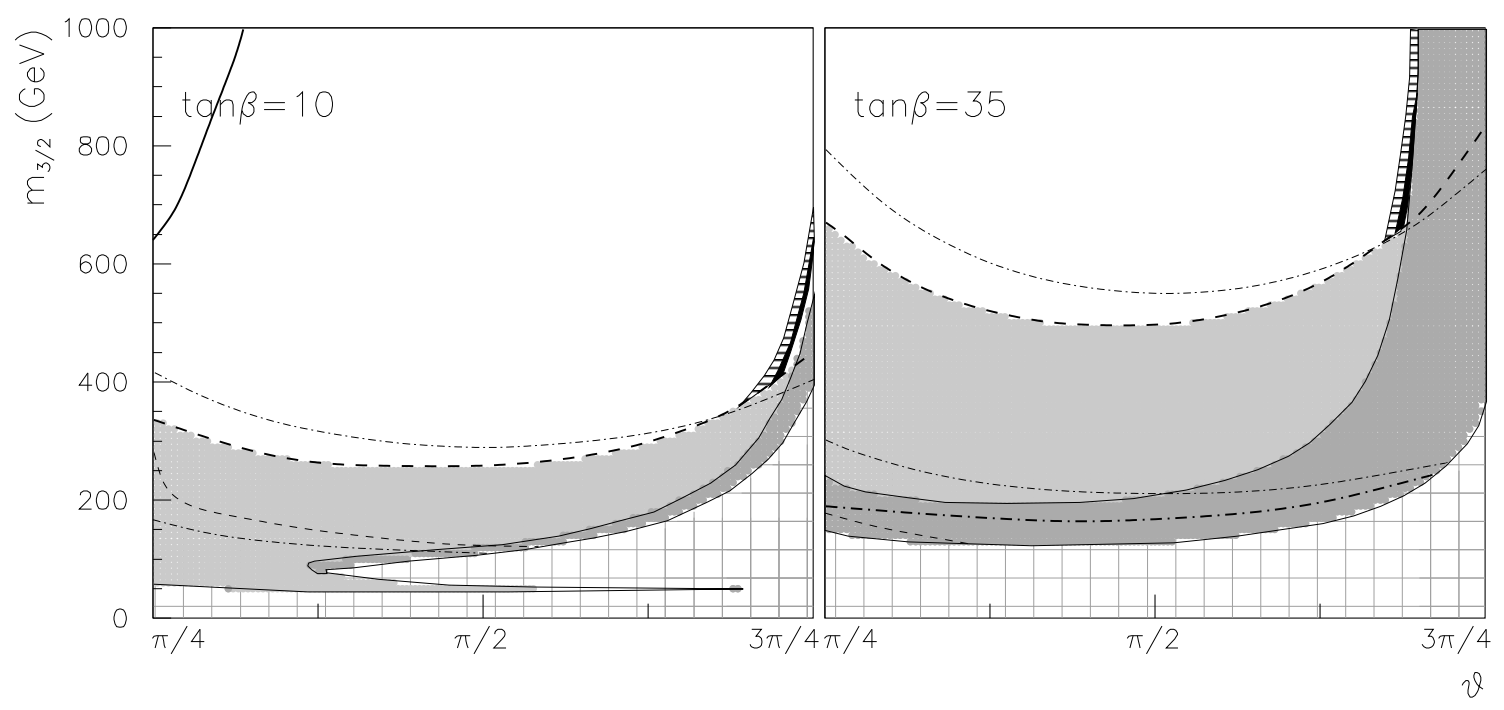

Figure 12: The same as Fig. 4 but for case C) in Table 1 and for $\tan \beta=10$ and 35 . Notice that for $\tan \beta=10$ the lightest stop is the LSP on the dark grey area whereas for $\tan \beta=35$ it is the lightest stau.

in $m_{L, E}^{2}$ due to the smaller modular weights. Similarly, the important decrease in the gluino mass implies a very light squark sector. This leads to a qualitatively different structure of the SUSY spectrum in which squarks and sleptons have a similar mass. This is illustrated in Fig. 11, where we have represented the resulting spectrum for 


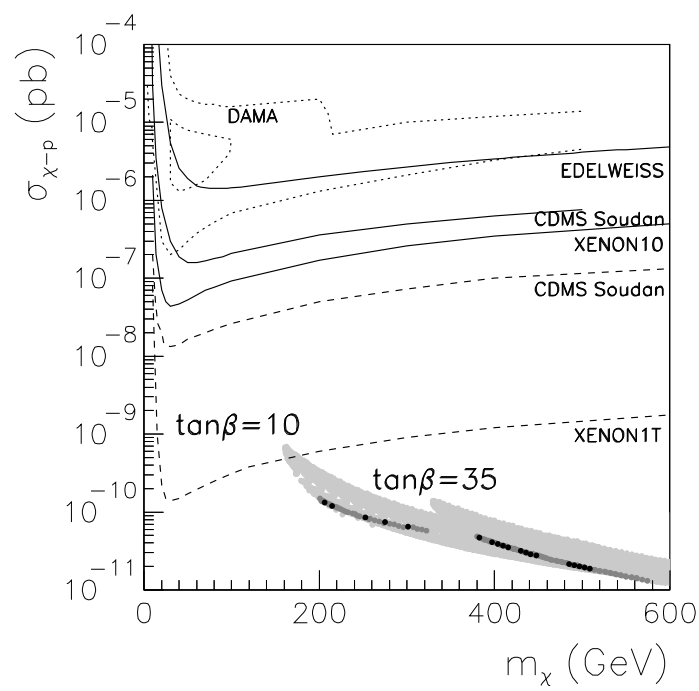

Figure 13: The same as Fig. 5 but for case C) in Table 1 and with $\tan \beta=10,35$.

$m_{3 / 2}=200 \mathrm{GeV}$ and $\tan \beta=10$ and 35 . Unlike the previous cases, this example displays very light gluinos and squarks. There are even regions where the stop is the LSP (especially for low values of $\tan \beta$ for which the top Yukawa is larger).

The regions allowed by experimental constraints become larger in this example, as we can see in Fig. 12, where the $\left(m_{3 / 2}, \theta\right)$ plane is depicted for $\tan \beta=10$ and 35 . It is important to mention that, due to the resulting light squarks, the supersymmetric contribution to $\mathrm{B}(b \rightarrow s \gamma)$ becomes sizable. Unlike in the previous examples, the experimental bound on this observable becomes the most stringent constraint, even for small values of $\tan \beta$. There are also areas which reproduce the correct dark matter relic density through coannihilation effects with the stop (for small values of $\tan \beta$ ) and the stau (for $\tan \beta \gtrsim 20$ ). Noticeably, in spite of the less negative modular weights for sleptons, the modifications in the RGEs (especially the decrease in $M_{1,2}$ ) imply smaller values for the Higgs mass parameters. In particular, $m_{H_{u}}^{2}$ is more negative, making it more difficult to avoid the UFB-3 constraint. Only for heavy gravitinos do allowed regions occur $\left(m_{3 / 2} \gtrsim 650 \mathrm{GeV}\right.$ is necessary for $\tan \beta=10$, whether for larger $\tan \beta$ gravitinos heavier than $1 \mathrm{TeV}$ are needed). As already observed in case $\mathrm{A}$ ), these regions never correspond to those with the correct neutralino relic density.

Finally, it is worth mentioning that, since the Higgs mass parameters have a smaller departure from universality, we do not expect large neutralino detection cross sections in this example. The results for $\sigma_{\tilde{\chi}_{1}^{0}-p}$ are represented in Fig. 13 for $\tan \beta=10$ and 35 and, clearly lie beyond the reach of current and projected direct dark matter searches. 

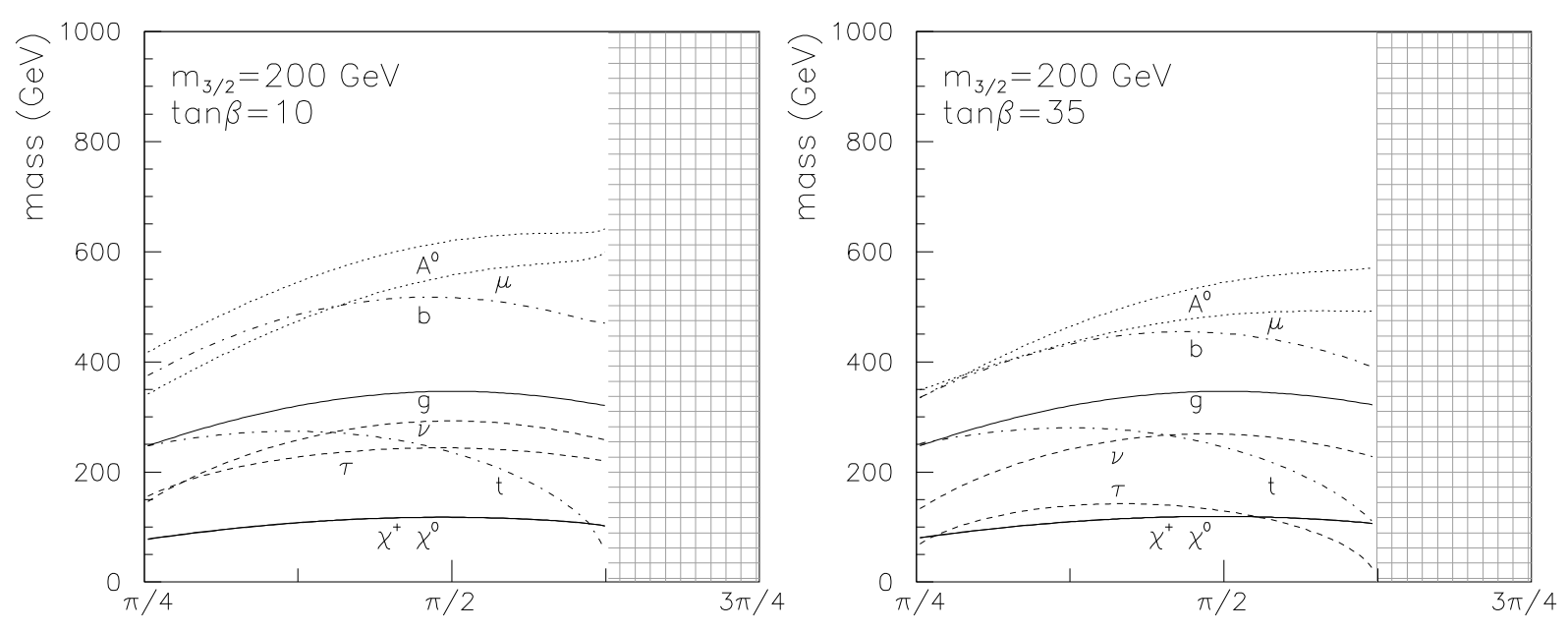

Figure 14: The same as Fig. 2 but for case D) in Table 1.

Another potentially interesting scenario is case D), once more due to the reduced modular weights for sleptons. As in the previous example, the region allowed at the GUT scale is $\theta \in[\pi / 4,3 \pi / 4],[5 \pi / 4,7 \pi / 4]$, where $m_{Q_{L}, L_{L}}^{2} \geq 0$. In this scenario one needs four extra multiplets, transforming like $(Q, \bar{Q}, D, \bar{D})$, and $\operatorname{Re} T=9$ for unification to take place, thus implying $b_{1}=-12, b_{2}=-4$, and $b_{3}=0$. The absence of running for $\alpha_{3}$ and therefore for the gluino mass parameters has the same consequences as in case $\mathrm{C}$ ), leading to a light squark spectrum. This is illustrated in Fig. 14, where the sparticle masses are plotted as a function of the Goldstino angle for $m_{3 / 2}=200$ $\mathrm{GeV}$ and $\tan \beta=10$ and 35. Interestingly, the running of the wino mass parameter is slightly enhanced and $M_{1} \sim M_{2}$ is found at the electroweak scale. This makes the lightest neutralino a mixed bino-wino state and almost degenerate in mass with the lightest chargino. Although this can lead to an increase of the resulting neutralino direct detection cross section, it also implies a more efficient neutralino annihilation and, consequently, a relic density which is too small to account for the dark matter of the Universe. In particular, one obtains $\Omega_{\tilde{\chi}_{1}^{0}} h^{2} \lesssim 0.01$ for the whole region with gravitinos lighter than $1 \mathrm{TeV}$, independently of the value of $\tan \beta$. Thus, although the area allowed by experimental constraint, represented in Fig. 15 for $\tan \beta=10$ and 35 is sizable, the astrophysical constraint on the relic density is never fulfilled. As in the previous examples, the presence of light squarks induce larger contributions to $\mathrm{B}(b \rightarrow s \gamma)$ and the experimental constraint on it excludes extensive regions of the parameter space, even at low $\tan \beta$. Furthermore, the steeper running of $M_{2}$ renders 


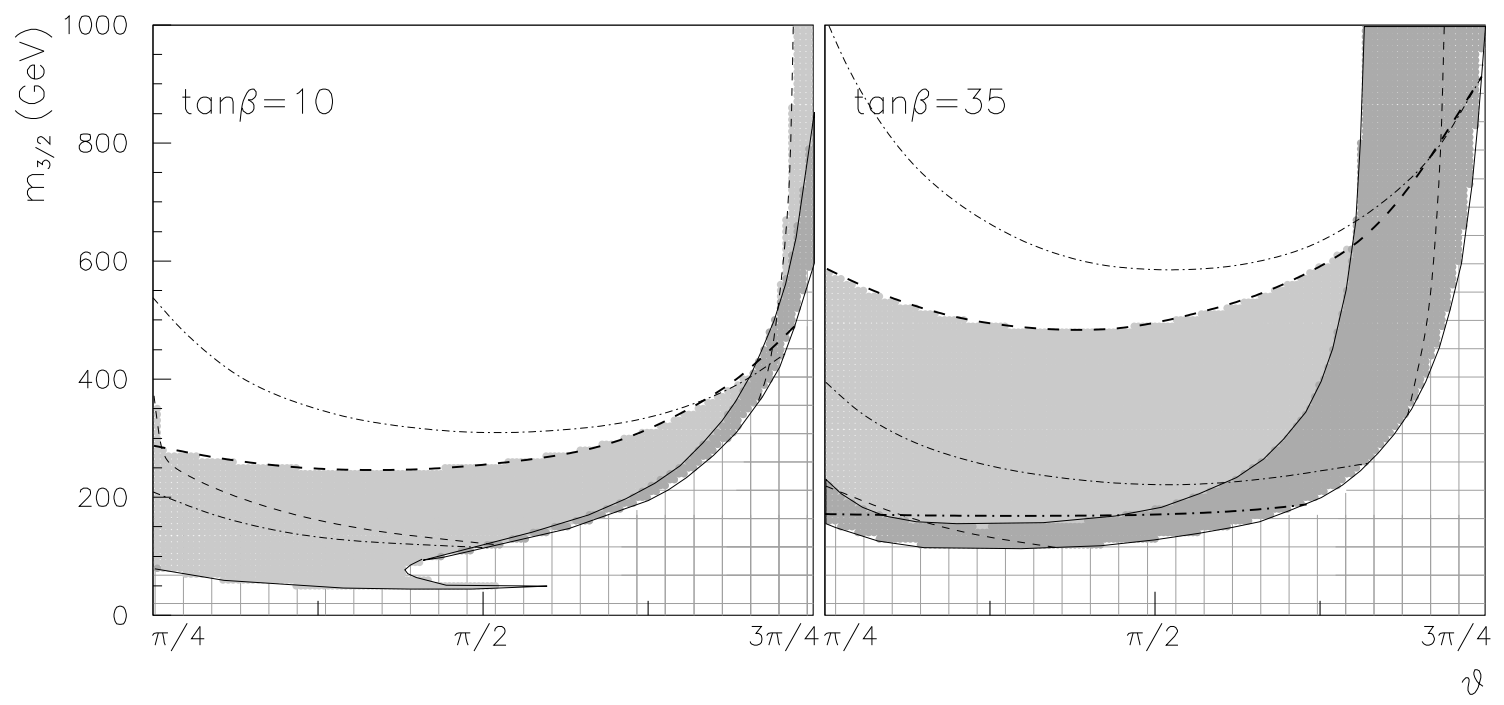

Figure 15: The same as Fig. 3 but for case D) in Table 1. Notice that for $\tan \beta=10$ the lightest stop is the LSP on the dark grey area whereas for $\tan \beta=35$ it is the lightest stau.

$m_{H_{u}}^{2}$ more negative, which makes the UFB constraints even more restrictive. All the area represented in Fig. 15 becomes excluded for this reason.

Finally, case E) in Table 1 corresponds to a $Z_{8}^{\prime}$ orbifold with a universal modulus, in which case $\operatorname{Re} T \sim 24$ is needed. Due to the small modular weights for sleptons this example yields similar results regarding the UFB constraints as case A), with allowed regions appearing only for very massive gravitinos and incompatible with the astrophysical bound on the dark matter relic density. Moreover, since the non-universality in the Higgs mass parameters $\left(m_{H_{u}}^{2}<m_{H_{d}}^{2}\right)$ is not the optimal to increase the neutralino detection cross section, the theoretical predictions for $\sigma_{\tilde{\chi}_{1}^{0}-p}$ are even smaller than those represented in Fig. 5.

The presence of different moduli can provide some extra freedom to the nonuniversalities of the soft scalar masses (3.9), which are then parametrized by new Goldstino angles, $\Theta_{i}$. We have analysed two scenarios of this kind, whose modular weights are described in Table 2, and which have also been shown to reproduce gauge coupling unification [50]. Scenario F) corresponds to a $Z_{6}$ orbifold with a rotated plane for which unification is achieved with $\operatorname{Re} T_{1} \sim 10 \gg \operatorname{Re} T_{2}$. In scenario G), a $Z_{2} \times Z_{2}$ orbifold was taken, again non-isotropic, with $T_{1} \gg T_{2,3}$.

In both examples, some combinations of the Goldstino angles lead to potentially interesting non-universalities in the soft scalar masses. In particular, it is always pos- 


\begin{tabular}{|c|ccccccc|}
\hline & $n_{Q_{L}}^{i}$ & $n_{u_{R}}^{i}$ & $n_{d_{R}}^{i}$ & $n_{e_{R}}^{i}$ & $n_{L_{L}}^{i}$ & $n_{H_{d}}^{i}$ & $n_{H_{u}}^{i}$ \\
\hline $\mathrm{F})$ & $0,-1,0$ & $\frac{-2}{3}, 0, \frac{-1}{3}$ & $0,-1,0$ & $\frac{-5}{3}, 0, \frac{-1}{3}$ & $\frac{-2}{3}, 0, \frac{-1}{3}$ & $0,0,-1$ & $0,0,-1$ \\
\hline $\mathrm{G})$ & $\frac{-1}{2}, \frac{-1}{2}, 0$ & $-1,0,0$ & $0,-1,0$ & $-1,0,0$ & $-1,0,0$ & $-1,0,0$ & $0,-1,0$ \\
\hline
\end{tabular}

Table 2: Modular weights for the scalar fields of two multimoduli heterotic orbifold scenarios that can reproduce gauge unification [50].

sible to enlarge the stau mass, thus avoiding UFB constraints. For instance, in case F) this can be done by choosing $\Theta_{3}^{2}=1$ (hence $\Theta_{1}^{2}=\Theta_{2}^{2}=0$ ) obtaining the following expressions for the soft terms,

$$
\begin{aligned}
m_{Q_{L}}^{2} & =m_{d_{R}}^{2}=m_{3 / 2}^{2}, \\
m_{u_{R}}^{2}=m_{e_{R}}^{2} & =m_{L_{L}}^{2}=m_{3 / 2}^{2}\left(1-\cos ^{2} \theta\right), \\
m_{H_{d}}^{2} & =m_{H_{u}}^{2}=m_{3 / 2}^{2}\left(1-3 \cos ^{2} \theta\right) .
\end{aligned}
$$

Alternatively, in case G) one can take $\Theta_{1}^{2}=0$ (and therefore $\Theta_{2}^{2}+\Theta_{3}^{2}=1$ ) and obtain

$$
\begin{aligned}
m_{Q_{L}}^{2} & =m_{3 / 2}^{2}\left(1-\frac{3}{2} \Theta_{2}^{2} \cos ^{2} \theta\right), \\
m_{u_{R}}^{2}=m_{e_{R}}^{2}=m_{L_{L}}^{2}=m_{H_{d}}^{2} & =m_{3 / 2}^{2} \\
m_{d_{R}}^{2}= & m_{H_{u}}^{2}=m_{3 / 2}^{2}\left(1-3 \Theta_{2}^{2} \cos ^{2} \theta\right) .
\end{aligned}
$$

Notice, however, that the resulting Higgs mass parameters are not adequate to obtain large neutralino detection cross sections. On the one hand, in case F) these are universal by construction since they have the same modular weights. On the other hand, in case G), the Higgs soft masses are related by $m_{H_{u}}^{2} / m_{H_{d}}^{2}=\left(1-3 \Theta_{2}^{2} \cos ^{2} \theta\right) /\left(1-3 \Theta_{1}^{2} \cos ^{2} \theta\right)$. If heavy staus are required, as in (3.22), $m_{H_{d}}^{2} \geq m_{H_{u}}^{2}$ is obtained, which implies small $\sigma_{\tilde{\chi}_{1}^{0}-p}$. In order to reproduce the optimal Higgs non-universality, $m_{H_{u}}^{2} \geq m_{H_{d}}^{2}$, one needs to take $\Theta^{2}>\Theta_{1}^{2}$ but then light staus appear (since $m_{e_{R}}^{2}=m_{L_{L}}^{2}=m_{H_{d}}^{2}$ ) which therefore lead to strong UFB constraints.

As a consequence, none of these two examples can provide larger theoretical predictions for $\sigma_{\tilde{\chi}_{1}^{0}-p}$ than those obtained in the optimised case of Fig. 9. 


\subsection{Non-thermal production of neutralinos from late gravitino decays}

Due to their extremely weak (gravitational) interactions, gravitinos can have a very long lifetime (typically longer than $10^{2}$ sec for $m_{3 / 2} \lesssim 1 \mathrm{TeV}$ ). Consequently, gravitinos which are produced thermally in the reheating phase after inflation and which decouple with a given relic density $\Omega_{3 / 2} h^{2}$ will decay at late times into the LSP (or cascading down to the LSP). This constitutes a source of non-thermal production of neutralinos, $\Omega_{\tilde{\chi}_{1}^{0}}^{N T P}$, which contributes to the total relic density of dark matter, and is related to the gravitino relic density as

$$
\Omega_{\tilde{\chi}_{1}^{0}}^{N T P} h^{2}=\frac{m_{\tilde{\chi}_{1}^{0}}}{m_{3 / 2}} \Omega_{3 / 2} h^{2} .
$$

It is easy, however to argue that $\Omega_{\tilde{\chi}_{1}^{0}}^{N T P}$ is typically very small in the orbifold models we have just presented. It can be shown that in the case of gravitinos that are much lighter than gluinos $\left(m_{3 / 2} \ll m_{\tilde{g}}\right)$, which generally holds in our case, the gravitino abundance from thermal production can be estimated as [67]

$$
\Omega_{3 / 2} h^{2} \approx 0.21\left(\frac{T_{R}}{10^{10} \mathrm{GeV}}\right)\left(\frac{100 \mathrm{GeV}}{m_{3 / 2}}\right)\left(\frac{m_{\tilde{g}}}{1 \mathrm{TeV}}\right)^{2} .
$$

The reheating temperature, $T_{R}$, is here a free parameter. The larger the reheating temperature, the more sizable $\Omega_{3 / 2} h^{2}$ becomes. However, in order not to reintroduce the infamous gravitino problem and spoil the results from Big Bang nucleosynthesis, the reheating temperature is constrained to be [68] $T_{R} \lesssim 10^{6} \mathrm{GeV}$ for a gravitino with a mass of order $m_{3 / 2} \approx 100 \mathrm{GeV}$. This implies an upper limit on the gravitino relic density, and thus on the non-thermal production of neutralinos. From (3.23) and (3.24), and using typical values for the gluino and neutralino mass, we obtain $\Omega_{\tilde{\chi}_{1}^{0}}^{N T P} \lesssim 2 \times 10^{-4}$. Thus, even if the reheating temperature was increased to $T_{R}=10^{8} \mathrm{GeV}$ (for heavier gravitinos, and provided their hadronic branching ratio is small), the contribution to the neutralino relic density is negligible. 


\section{Modifications due to the presence of an anoma- lous $U(1)$}

As discussed in the Introduction, an anomalous $U(1)$ [28] is usually present after compactification $^{7}$. For example, it was found in [71] that only 192 different three-generation models containing the $S U(3) \times S U(2) \times U(1)^{n}$ gauge group can be constructed within the $Z_{3}$ orbifold with two Wilson lines. The matter content of 175 of them was analysed in detail and only 7 of them turn out not to have an anomalous $U(1)$ associated. Let us recall that the presence of the anomalous $U(1)$ is crucial for model building [29]. It generates a FI contribution to the D-term [28], breaking extra $U(1)$ symmetries, and allowing the construction of realistic standard-like models [30-32,35-38]. Let us now discuss the possible contributions to soft scalar masses generated because of the FI breaking, and their effect on the neutralino direct detection cross section.

The FI breaking induces additional terms to soft SUSY-breaking scalar masses ${ }^{8}$ due to F-terms, namely, the so-called D-term contribution [39-44]. In particular, the presence of an anomalous $U(1)$ after compactification generates the dilaton-dependent FI term. That is, the D-term of the anomalous $U(1)$ is given by

$$
D^{A}=\frac{\delta_{G S}^{A}}{S+S^{*}}+\sum_{\alpha}\left(T+T^{*}\right)^{n_{\alpha}} q_{\alpha}^{A}\left|\phi_{\alpha}\right|^{2}
$$

where the first term corresponds to the dilaton-dependent Fayet-Iliopoulos term with the GS coefficient $\delta_{G S}^{A}$ proportional to the value of the anomaly, and the second one is the usual D-term with the $U(1)$ charges $q_{\alpha}^{A}$ of the fields $\phi_{\alpha}$. Then, some of these fields (with vanishing hypercharges), let us call them $C_{\beta}$, develop large vacuum expectation values (VEVs) along the D-flat direction in order to cancel the FI term, inducing the D-term contribution to the soft scalar masses of the observable fields. The resulting scalar mass squared is given by $[41]^{9}$

$$
m_{\alpha}^{2}=m_{3 / 2}^{2}\left\{1+n_{\alpha} \cos ^{2} \theta+q_{\alpha}^{A} \frac{\sum_{\beta}\left(T+T^{*}\right)^{n_{\beta}} q_{\beta}^{A}\left|C_{\beta}\right|^{2}\left[\left(6-n_{\beta}\right) \cos ^{2} \theta-5\right]}{\sum_{\beta}\left(T+T^{*}\right)^{n_{\beta}}\left(q_{\beta}^{A}\right)^{2}\left|C_{\beta}\right|^{2}}\right\},
$$

\footnotetext{
${ }^{7}$ In [70], some conditions for the absence of the anomalous $U(1)$ are discussed, and classifications of models with anomalous $U(1)$ are also attempted.

${ }^{8}$ Let us remark that there are no additional contributions to gaugino masses and A-terms when Higgs fields relevant to such symmetry breaking have less F-term than those of dilaton and moduli fields.

${ }^{9}$ Assuming for simplicity that the fields $C_{\beta}$ have no other $U(1)$ charges.
} 


\begin{tabular}{|c|ccc|}
\hline & $q_{L_{L}, e_{r}}^{A} / q_{C}^{A}$ & $q_{H_{d}}^{A} / q_{C}^{A}$ & $q_{H_{u}}^{A} / q_{C}^{A}$ \\
\hline $\mathrm{A}-\mathrm{I})$ & -2 & 0 & 0 \\
\hline $\mathrm{A}-\mathrm{II})$ & -2 & 0 & -2 \\
\hline $\mathrm{A}-\mathrm{III})$ & -2 & $\frac{1}{2}$ & -2 \\
\hline
\end{tabular}

Table 3: Variations of scenario A) in Table 1. The fields have the same modular weights, but we have assigned the above $U(1)$ charges to the slepton and Higgs fields.

where the first two terms are the usual contributions (see (3.11)), and the third term is the D-term contribution. Obviously, if the observable fields have vanishing $U(1)$ charges, $q_{\alpha}^{A}$, this contribution is also vanishing, and we recover the situation of the previous Section. However, the observable fields have usually non-vanishing charges in explicit models [30-38], and the effect of this contribution must be taken into account in the analysis.

As we can see in the above formula, the D-term contribution generates in general an additional non-universality among soft scalar masses which depends on $q_{\alpha}^{A}$. Let us simplify the analysis considering the case where only a single field $C$ develops a VEV in order to cancel the FI term. Thus the above result reduces to the following form:

$$
m_{\alpha}^{2}=m_{3 / 2}^{2}\left\{1+n_{\alpha} \cos ^{2} \theta+\frac{q_{\alpha}^{A}}{q_{C}^{A}}\left[\left(6-n_{C}\right) \cos ^{2} \theta-5\right]\right\},
$$

where $q_{C}^{A}$ and $n_{C}$ are the $U(1)$ charge and modular weight of the field $C$, respectively. It is worth emphasizing here that even in the dilaton-dominated case $(\cos \theta=0)$ the soft scalar masses are non-universal,

$$
m_{\alpha}^{2}=m_{3 / 2}^{2}\left(1-5 \frac{q_{\alpha}^{A}}{q_{C}^{A}}\right) .
$$

This result should be compared with the one in (3.11). The contributions $-5 q_{\alpha}^{A} / q_{C}^{A}$ correspond to the $\delta$ 's in (2.1) and (2.2). It is noteworthy that, contrary to the cases without an anomalous $\mathrm{U}(1)$ analysed in the previous Section, positive values for the non-universalities are now possible, by choosing $q_{\alpha}^{A} / q_{C}^{A}<0$. This is welcome, as we will see, in order to enhance the stau masses and thus avoid the UFB constraints, or increase the value of $m_{H_{u}}^{2}$. Moreover, we may expect that, for appropriate values of the $U(1)$ charges, the above additional terms lead to interesting values for the neutralino detection cross section.

Let us consider again scenario A) of the previous section, defined by expressions (3.15) and (3.16), where the sleptons masses were too small and therefore problematic. 

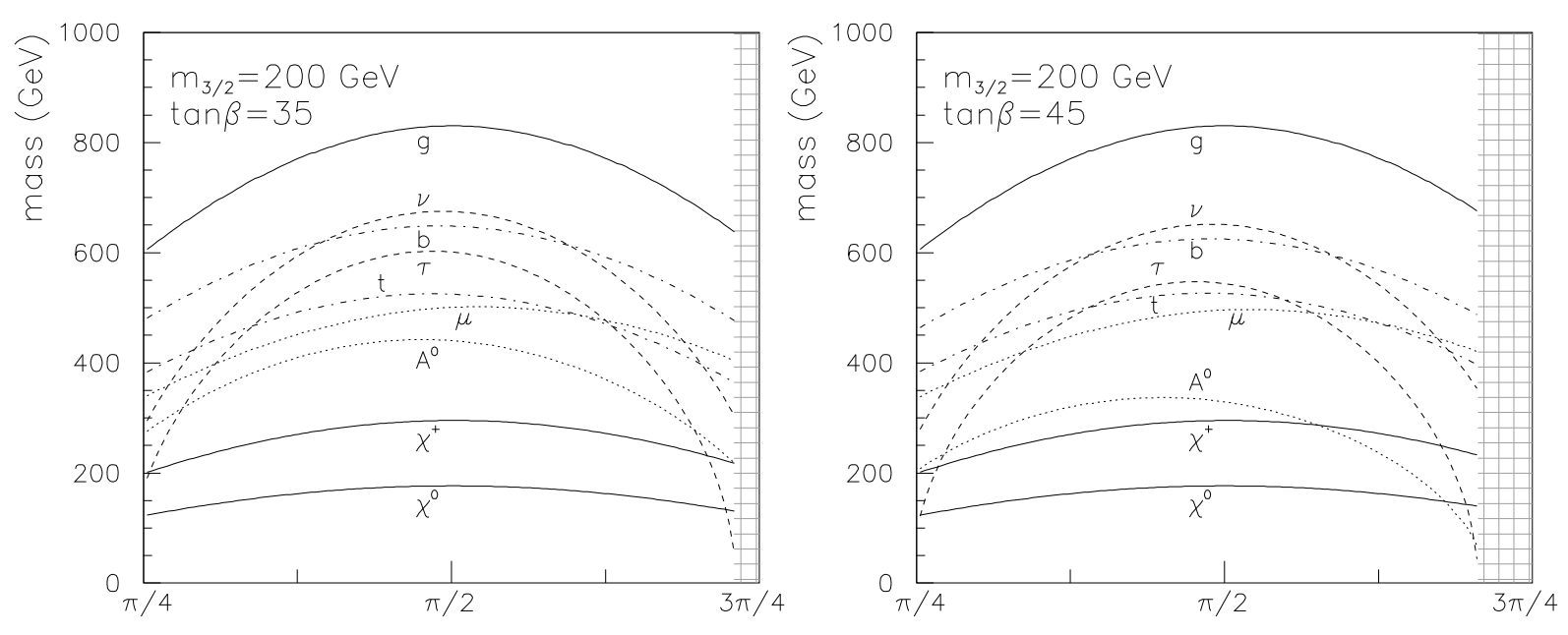

Figure 16: The same as Fig. 2 but for example A-I) of Table. 3 with $\tan \beta=35$ and 45 . Soft masses satisfy (3.15) and (3.16) with slepton masses modified according to Eq.(4.30).

Using (4.27), these are now given by

$$
m_{L_{L}, e_{R}}^{2}=m_{3 / 2}^{2}\left\{1-3 \cos ^{2} \theta+\frac{q_{L_{L}, e_{R}}^{A}}{q_{C}^{A}}\left[\left(6-n_{C}\right) \cos ^{2} \theta-5\right]\right\} .
$$

For instance, assuming that $C$ is a twisted field with modular weight $n_{C}=-2$, and the $\operatorname{ratios}^{10} q_{L_{L}, e_{R}}^{A} / q_{C}^{A}=-2$, one has

$$
m_{L_{L}, e_{R}}^{2}=m_{3 / 2}^{2}\left(11-19 \cos ^{2} \theta\right)
$$

This corresponds to case A-I) in Table 3. The degree of non-universality, as defined in (2.2), is therefore $\delta_{L_{L}, e_{R}}=10-19 \cos ^{2} \theta$. Unlike the examples in the previous section, where all the $\delta$ 's had to be negative, positive values can now be obtained.

Condition $\cos ^{2} \theta \leq 1 / 3$ is no longer necessary in order to avoid tachyonic sleptons, since $\cos ^{2} \theta \leq 11 / 19$ is sufficient, thus implying broader allowed regions. Noticeably, unlike the examples in the previous Section, gaugino masses (3.17) can be of the same order, and even smaller than slepton masses at the GUT scale. For example, with $\cos ^{2} \theta=1 / 2$ one obtains $m_{L_{L}, e_{R}}^{2}=\frac{3}{2} m_{3 / 2}^{2}$, with gaugino masses of the same order

\footnotetext{
${ }^{10}$ These values for modular weights, and ratios between $U(1)$ charges of observable and FI fields, are typically obtained in explicit models [30-32].
} 
$M_{a}^{2} \simeq m_{L_{L}, e_{R}}^{2}$. And in the dilaton dominated limit one obtains $m_{L_{L}, e_{R}}^{2}=11 m_{3 / 2}^{2}$, much larger than gaugino masses, for which $M_{a}^{2} \simeq 3 m_{3 / 2}^{2}$.

Very heavy sleptons are therefore possible and, although their masses still decrease away from the dilaton limit, in most of the parameter space their mass is similar to that of squarks. Such a heavy scalar sector is clearly shown in Fig. 16 for $m_{3 / 2}=200$ $\mathrm{GeV}$ and $\tan \beta=35$ and 45. As we can see, the lightest neutralino and chargino are much lighter and the lightest stau rarely becomes the LSP. Only along extremely narrow areas, which appear for $\tan \beta \gtrsim 25$ at the edge of the allowed zones, is the stau lighter than the neutralino. Consequently, the allowed parameter space where the lightest neutralino is the LSP is more extensive and larger values of $\tan \beta$ are allowed. This leads to a more efficient decrease of both the pseudoscalar and heavy scalar Higgs masses.

The resulting $\left(m_{3 / 2}, \theta\right)$ parameter space is represented in Fig. 17 for $\tan \beta=35,45$. The most salient consequence of the increase in the slepton masses is the reduction of the regions excluded by the UFB-3 constraint (this is particularly true in the dilaton limit, as pointed out by [72]). Contrary to what happens in case A), for which the whole parameter space was excluded (see Fig. 4), now only some points with $m_{3 / 2} \lesssim 100 \mathrm{GeV}$ are disfavoured. On the other hand, as a consequence of the increase in $\tan \beta$ and the associated decrease of the pseudoscalar Higgs mass, the experimental bounds on $B_{s}^{0} \rightarrow \mu^{+} \mu^{-}$and $b \rightarrow s \gamma$ become much more constraining. These imply a stronger lower limit on the gravitino mass as we can see in the plot.

Regarding the neutralino relic density, for $\tan \beta \lesssim 25$, the stau coannihilation regions are not present and $\Omega_{\tilde{\chi}_{1}^{0}} h^{2} \gtrsim 0.3$ is obtained in the whole $\left(m_{3 / 2}, \theta\right)$ plane. The coannihilation strip only appears for $\tan \beta \gtrsim 25$, where the correct relic density can be reproduced in extremely narrow areas. However, the most interesting case is $\tan \beta \gtrsim 30$, where thanks to the decrease in the pseudoscalar Higgs mass, we can find regions in the parameter space where $2 m_{\tilde{\chi}_{1}^{0}} \approx m_{A}$, in which case the resonant annihilation of neutralinos helps reproducing the WMAP results. Due to this effect, the regions of the parameter space satisfying experimental and astrophysical constraints are now significantly larger. For instance, with $\tan \beta=35$ we find allowed points for the whole range of gravitino masses between $350 \mathrm{GeV}$ to $1000 \mathrm{GeV}$. Notice also that in the dilaton limit, $\theta=\pi / 2$, the region reproducing the WMAP relic density is experimentally excluded for $\tan \beta \lesssim 47$.

The decrease on the Higgs masses for $\tan \beta \gtrsim 35$ leads to an enhancement of the neutralino-nucleon cross section. However, for such values of $\tan \beta$ large branching 


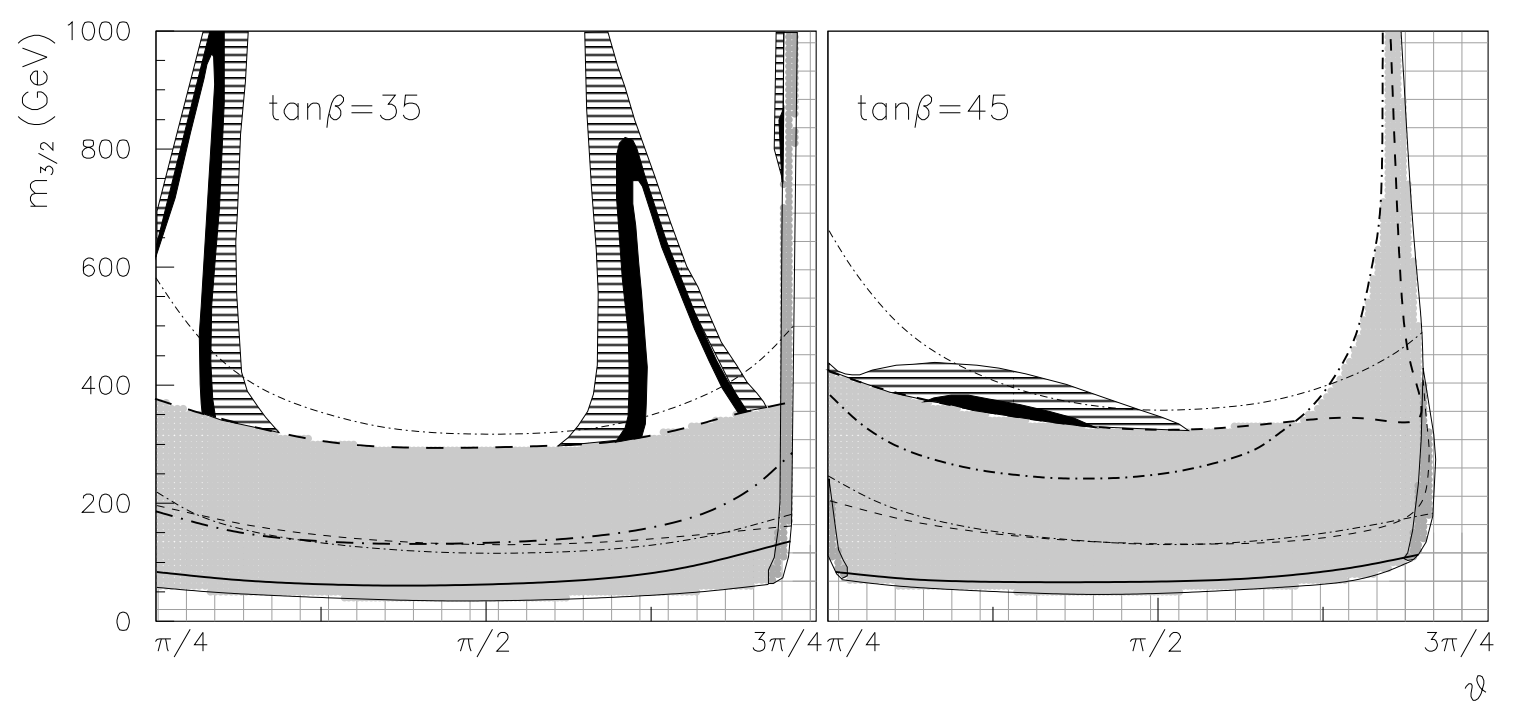

Figure 17: The same as Fig. 3 but for example A-I) of Table. 3 with $\tan \beta=35$, 45. Soft masses satisfy (3.15) and (3.16) with slepton masses modified according to Eq.(4.30).
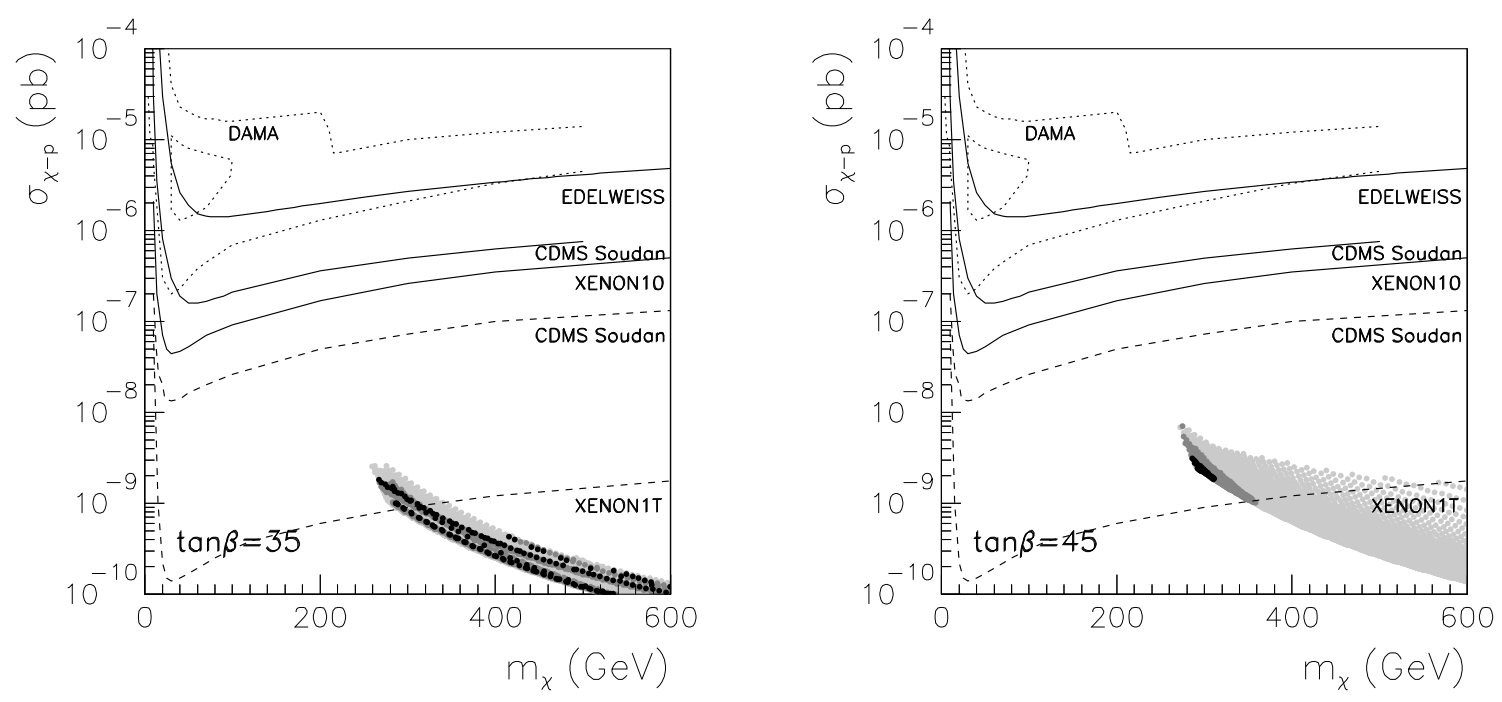

Figure 18: The same as Fig. 5 but for example A-I) of Table. 3 with $\tan \beta=35$, 45. Soft masses satisfy (3.15) and (3.16) with slepton masses modified according to Eq.(4.30).

ratios for the rare processes $b \rightarrow s \gamma$ and $B_{s}^{0} \rightarrow \mu^{+} \mu^{-}$are found, which therefore put stringent upper bounds on $\sigma_{\tilde{\chi}_{1}^{0}-p}$. This can be seen in Fig. 18, where the theoretical predictions for the neutralino detection cross section are represented as a function of the neutralino mass for $\tan \beta=35,45$. The cross section is bounded to be $\sigma_{\tilde{\chi}_{1}^{0}-p} \lesssim 2 \times 10^{-9} \mathrm{pb}$ by the aforementioned constraints. The corresponding values for 

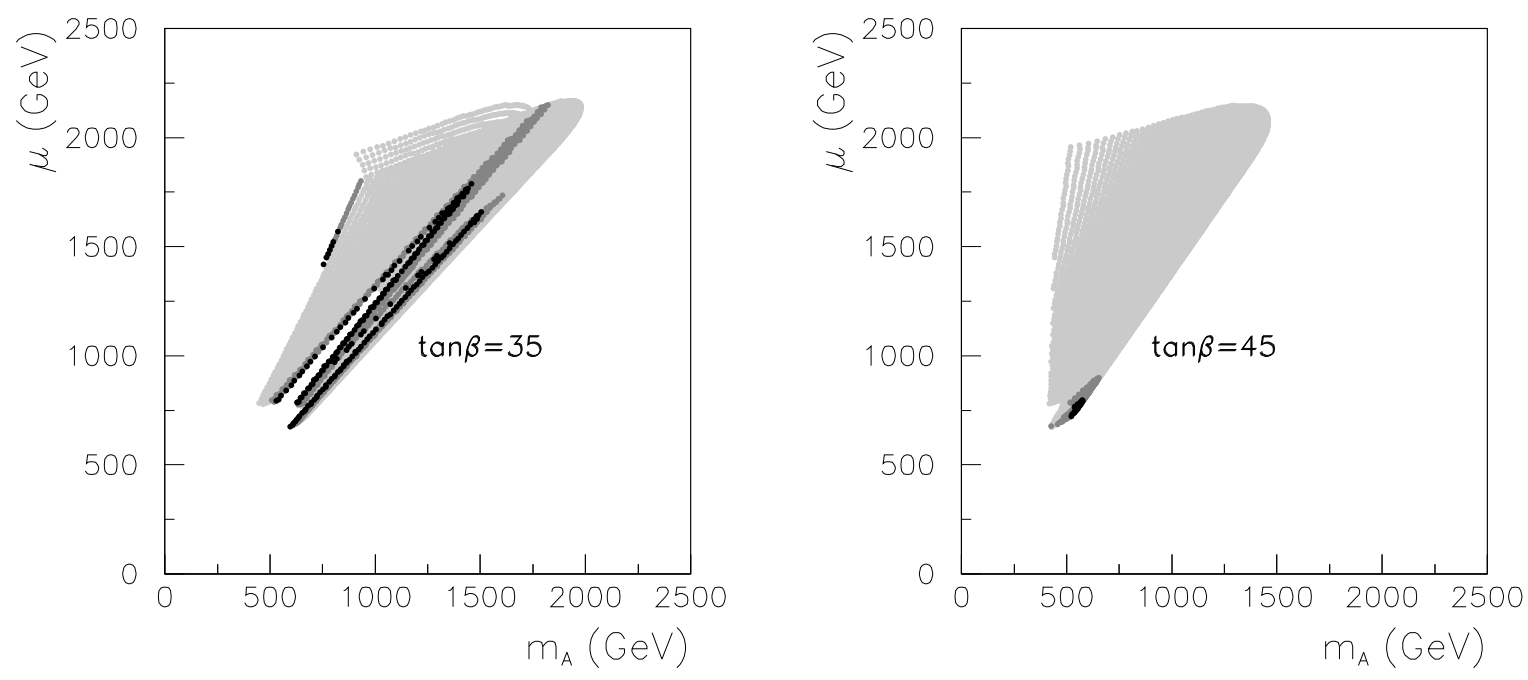

Figure 19: The same as in Fig. 6 but for example A-I) of Table. 3 with $\tan \beta=35,45$. Soft masses satisfy (3.15) and (3.16) with slepton masses modified according to Eq.(4.30).

$\mu$ and pseudoscalar mass are shown in Fig. 19. Both are rather large, comparable to what we obtained in Fig. 10 for the optimised case in the previous Section. For such large $\tan \beta$ the small CP-odd Higgs mass often leads to an excessive contribution to $B_{s}^{0} \rightarrow \mu^{+} \mu^{-}$, incompatible with the present experimental bound.

In order to further increase the neutralino-nucleon cross section while avoiding the experimental constraints, we need to find a way to decrease the Higgs masses even for small values of $\tan \beta$. This can be done by further exploiting the D-term contribution if we assume that also the Higgses have non-vanishing anomalous $U(1)$ charge. For example, setting $q_{H_{u}}^{A} / q_{C}^{A}=-2$, using result (4.27) equation (3.16) is modified as

$$
\begin{aligned}
& m_{H_{u}}^{2}=m_{3 / 2}^{2}\left(11-17 \cos ^{2} \theta\right), \\
& m_{H_{d}}^{2}=m_{3 / 2}^{2}\left(1-3 \cos ^{2} \theta\right) .
\end{aligned}
$$

We have labelled this as case A-II) in Table 3. The degree of non-universality, using notation (2.1), is given by $\delta_{H_{u}}=10-17 \cos ^{2} \theta$ and $\delta_{H_{d}}=-3 \cos ^{2} \theta$. In the dilatondominated case this turns out to be very large, $\delta_{H_{u}}=10$. Notice once more that, due to the presence of the anomalous $\mathrm{U}(1)$, positive values for $\delta_{H_{u}}$ can be obtained.

Due to the large non-universalities, the $\mu$ parameter and heavy Higgs masses can be significantly reduced, even for moderate values of $\tan \beta$, and especially in the dilaton limit. This is evidenced in Fig. 20 where the SUSY spectrum is plotted for $m_{3 / 2}=$ $200 \mathrm{GeV}$ and $\tan \beta=10$ and 25 . The lightest neutralino has an increased Higgsino 

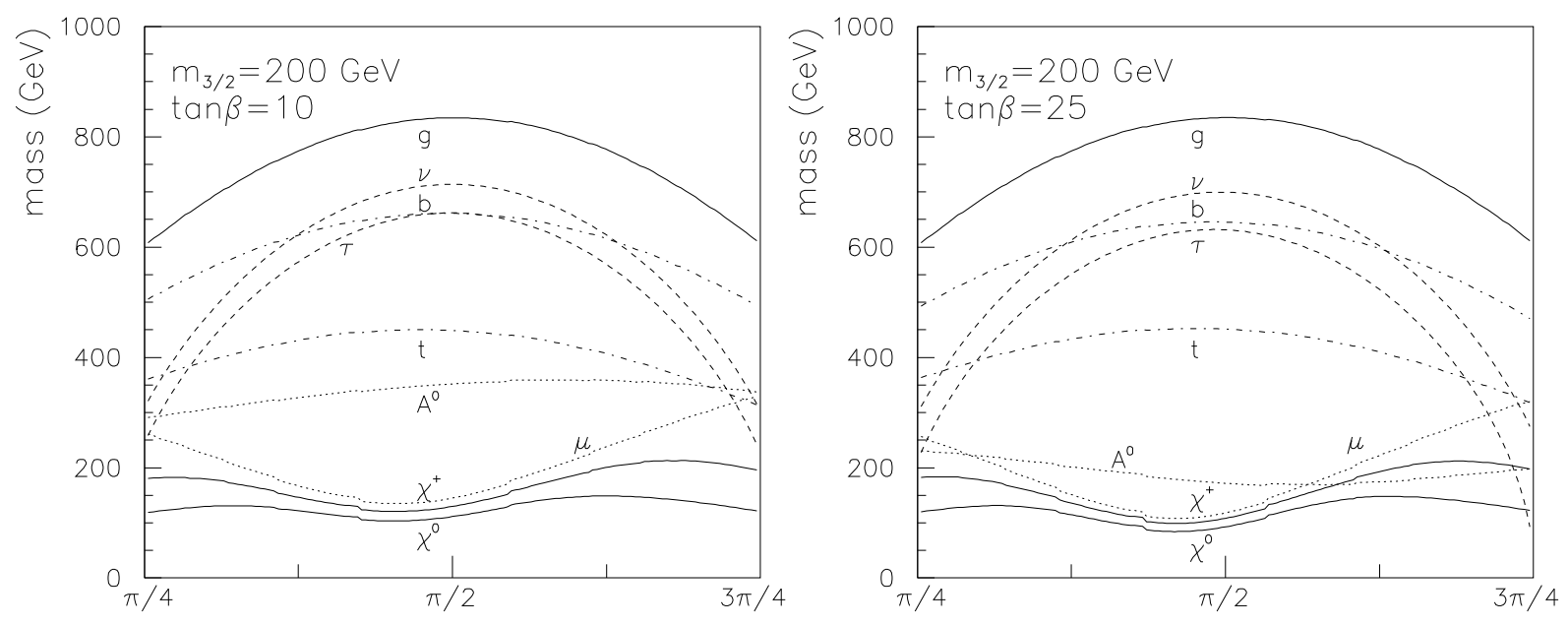

Figure 20: The same as Fig. 2 but for example A-II) of Table. 3 with $\tan \beta=10$ and 25 . Soft masses satisfy (3.15) with slepton masses modified according to Eq.(4.30) and Higgs mass parameters given by $(4.31)$.

composition in the area close to the dilaton-dominated limit and its mass is similar to that of the lightest chargino. This, together with the decrease in $m_{A^{0}}$, leads to a more effective neutralino annihilation in the early Universe and hence a reduced relic density towards $\theta=\pi / 2$.

The associated $\left(m_{3 / 2}, \theta\right)$ plane is represented in Fig. 21 for $\tan \beta=10$ and 25 . Extensive areas of the parameter space become excluded since $m_{A}^{2}$ becomes negative. These regions correspond to the ruled areas above the experimentally allowed ones. They are specially constraining in the dilaton limit, for which $m_{3 / 2} \lesssim 300 \mathrm{GeV}$ is needed. As $\tan \beta$ increases the dilaton limit eventually becomes excluded and the allowed regions shrink towards $\theta=\pi / 4$ and $3 \pi / 4$. As mentioned above, the resulting neutralino relic density is too small in the dilaton limit, but away from it, regions appear where the WMAP result is reproduced. Once more, due to the increase of the stau mass and $m_{H_{u}}^{2}$, the UFB constraints pose no problem in this scenario, and all the points depicted in Fig. 21 satisfy them.

The decrease of the $\mu$ parameter and the resulting larger Higgsino component for the lightest neutralino, $N_{13}^{2}+N_{14}^{2} \approx 0.4$, contribute to the increase of $\sigma_{\tilde{\chi}_{1}^{0}-p}$. The neutralinonucleon cross section is depicted on the left-hand side of Fig. 22 for $\tan \beta=25$, displaying neutralinos with $\sigma_{\tilde{\chi}_{1}^{0}-p} \gtrsim 10^{-7} \mathrm{pb}$ and a mass in the range $m_{\tilde{\chi}_{1}^{0}} \approx 200-500 \mathrm{GeV}$ 


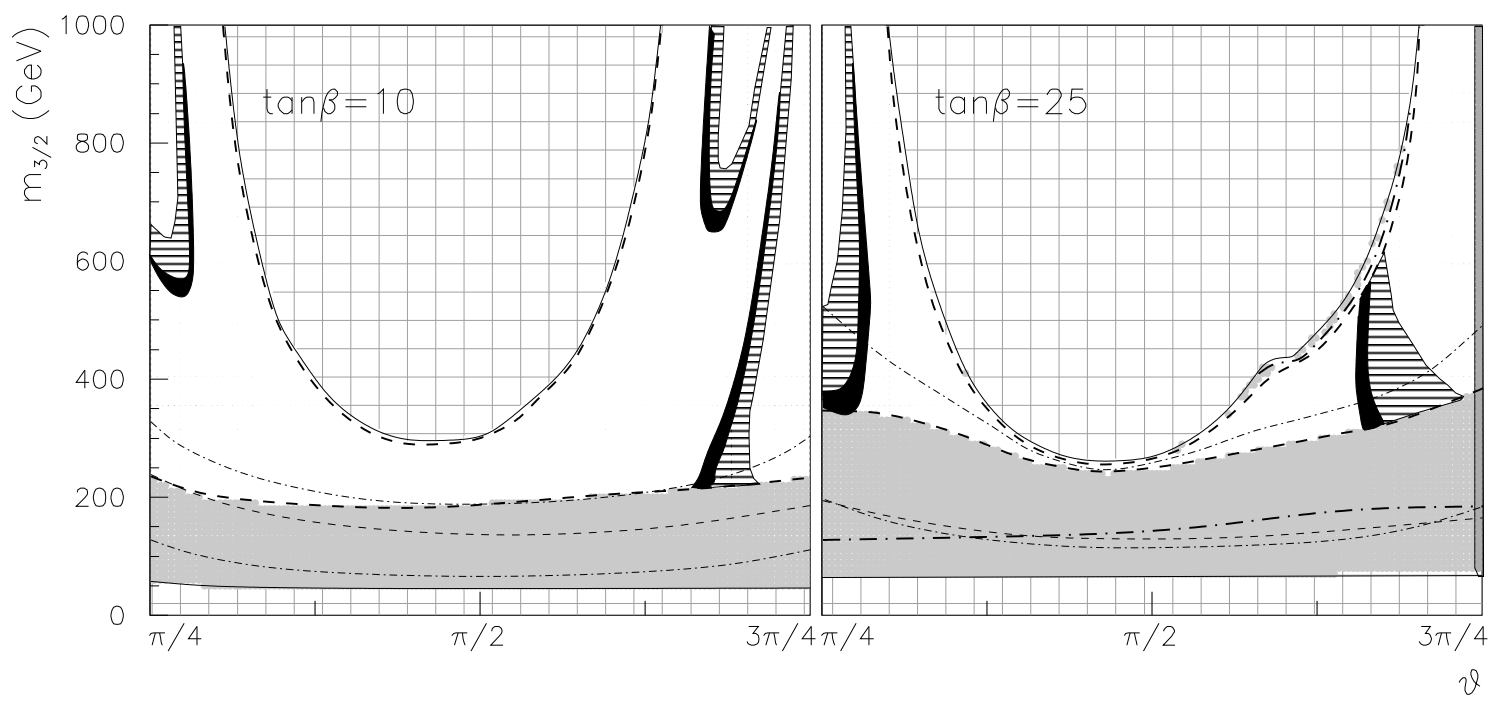

Figure 21: The same as Fig. 3 but for example A-II) of Table. 3 with $\tan \beta=10$ and 25 . Soft masses satisfy (3.15) with slepton masses modified according to Eq.(4.30) and Higgs mass parameters given by (4.31).
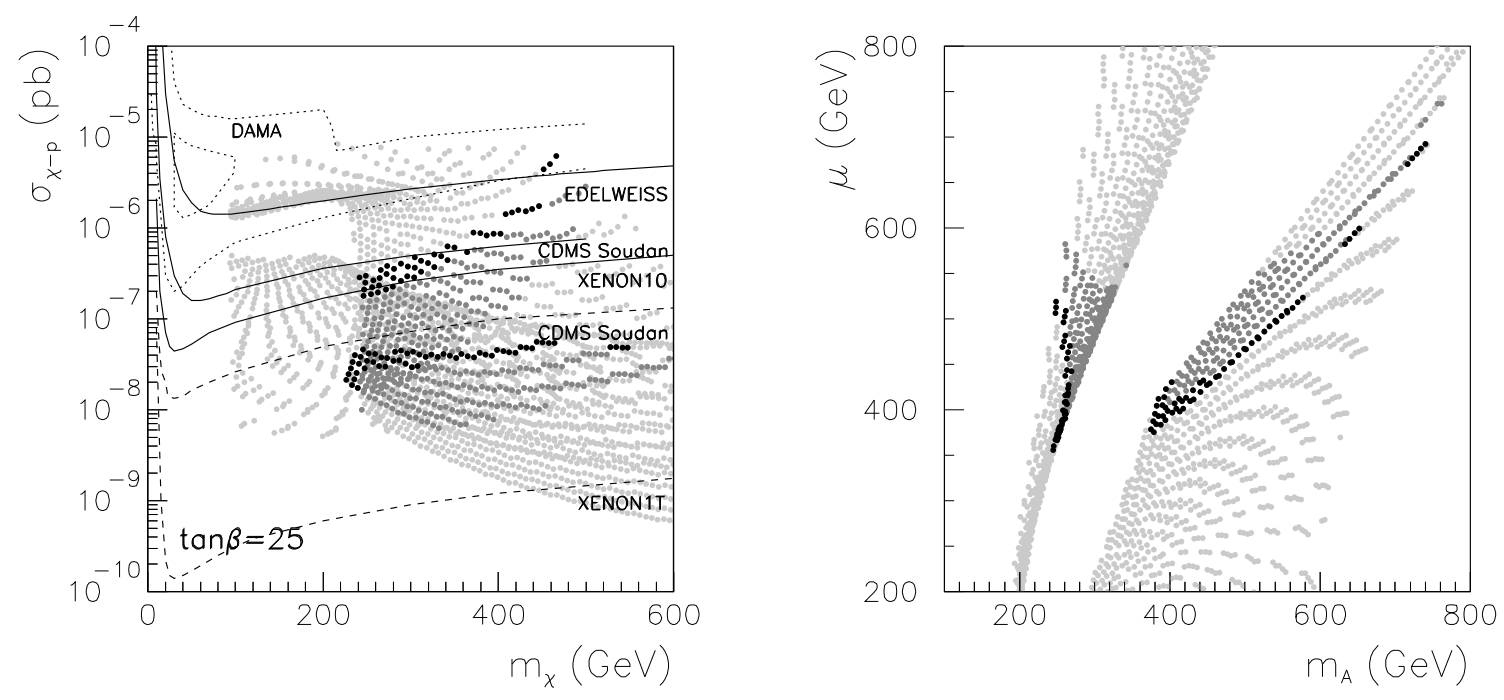

Figure 22: The same as Fig. 5 but for example A-II) of Table. 3 with $\tan \beta=25$. Soft masses satisfy (3.15) with slepton masses modified according to Eq.(4.30) and Higgs mass parameters given by (4.31).

that would be within the reach of the CDMS Soudan or XENON10 experiments. On the right-hand side of Fig. 22 the corresponding value of the $\mu$ parameter is represented as a function of the pseudoscalar Higgs mass for the same example, evidencing the de- 
crease in both quantities. The points with a larger detection cross section correspond to those with $m_{A} \approx 220-240 \mathrm{GeV}$ and $\mu \approx 350-500 \mathrm{GeV}$.

Notice that, unlike the previous case, a significant increase of $\sigma_{\tilde{\chi}_{1}^{0}-p}$ is obtained while keeping the observables $\mathrm{B}(b \rightarrow s \gamma)$ and $\mathrm{B}\left(B_{s}^{0} \rightarrow \mu^{+} \mu^{-}\right)$under control. As already mentioned, this owes to the fact that large values of $\tan \beta$ are no longer needed for obtaining sizable detection cross sections.

The Higgs non-universality can be further increased if we also consider $H_{d}$ to be charged under the anomalous $U(1)$. For example, taking $q_{H_{d}}^{A} / q_{C}^{A}=1 / 2$, which corresponds to case A-III) in Table 3, the soft masses for the Higgses become

$$
\begin{aligned}
& m_{H_{u}}^{2}=m_{3 / 2}^{2}\left(11-17 \cos ^{2} \theta\right), \\
& m_{H_{d}}^{2}=m_{3 / 2}^{2}\left(-\frac{3}{2}+\cos ^{2} \theta\right) .
\end{aligned}
$$

In this case the non-universalities are given by $\delta_{H_{u}}=10-17 \cos ^{2} \theta$, and $\delta_{H_{d}}=-\frac{5}{2}+$ $\cos ^{2} \theta$, which implies that in the dilaton limit they become $\delta_{H_{u}}=10$ and $\delta_{H_{d}}=-\frac{5}{2}$.

The associated parameter space is represented in Fig. 23 for $\tan \beta=20$. As in the previous example, extensive areas of the $\left(m_{3 / 2}, \theta\right)$ plane are ruled out because of $m_{A}^{2}$ becoming negative. Now, the region around the dilaton-dominated case, where the non-universality is maximal, is completely excluded for this reason. Only narrow allowed areas of the parameter space for specific values of the Goldstino angle survive. These allowed areas become smaller as $\tan \beta$ increases, since the pseudoscalar mass becomes tachyonic more easily, and eventually disappear for $\tan \beta \gtrsim 30$.

As a consequence of the further decrease in $m_{H_{d}}^{2}$, even smaller values of $\tan \beta$ are enough in order to obtain light pseudoscalar and scalar Higgses. Consequently, values of $\sigma_{\tilde{\chi}_{1}^{0}-p}$ within the reach of present experiments can now be found for even smaller values of $\tan \beta$. These are shown as a function of the neutralino mass on the lefthand side of Fig. 24 for $\tan \beta=20$, which is the optimal choice for this case. The pseudoscalar mass is very small in these points, as evidenced on the right-hand side of Fig. 24, where the $\mu$ parameter is plotted versus $m_{A}$. The points with $\sigma_{\tilde{\chi}_{1}^{0}-p} \gtrsim 10^{-7} \mathrm{pb}$ correspond to those with $m_{A} \lesssim 200 \mathrm{GeV}$, and have a moderate Higgsino composition.

Larger values of $q_{H_{d}}^{A} / q_{C}^{A}$ would further reduce $m_{H_{d}}^{2}$ and lead to more sizable nonuniversalities. However, this makes it more difficult to obtain positive $\mu^{2}$ or $m_{A}^{2}$, and the allowed areas in the parameter space become more fine-tuned. For instance, with $q_{H_{d}}^{A} / q_{C}^{A}=1$, which leads to

$$
m_{H_{u}}^{2}=m_{3 / 2}^{2}\left(11-17 \cos ^{2} \theta\right)
$$




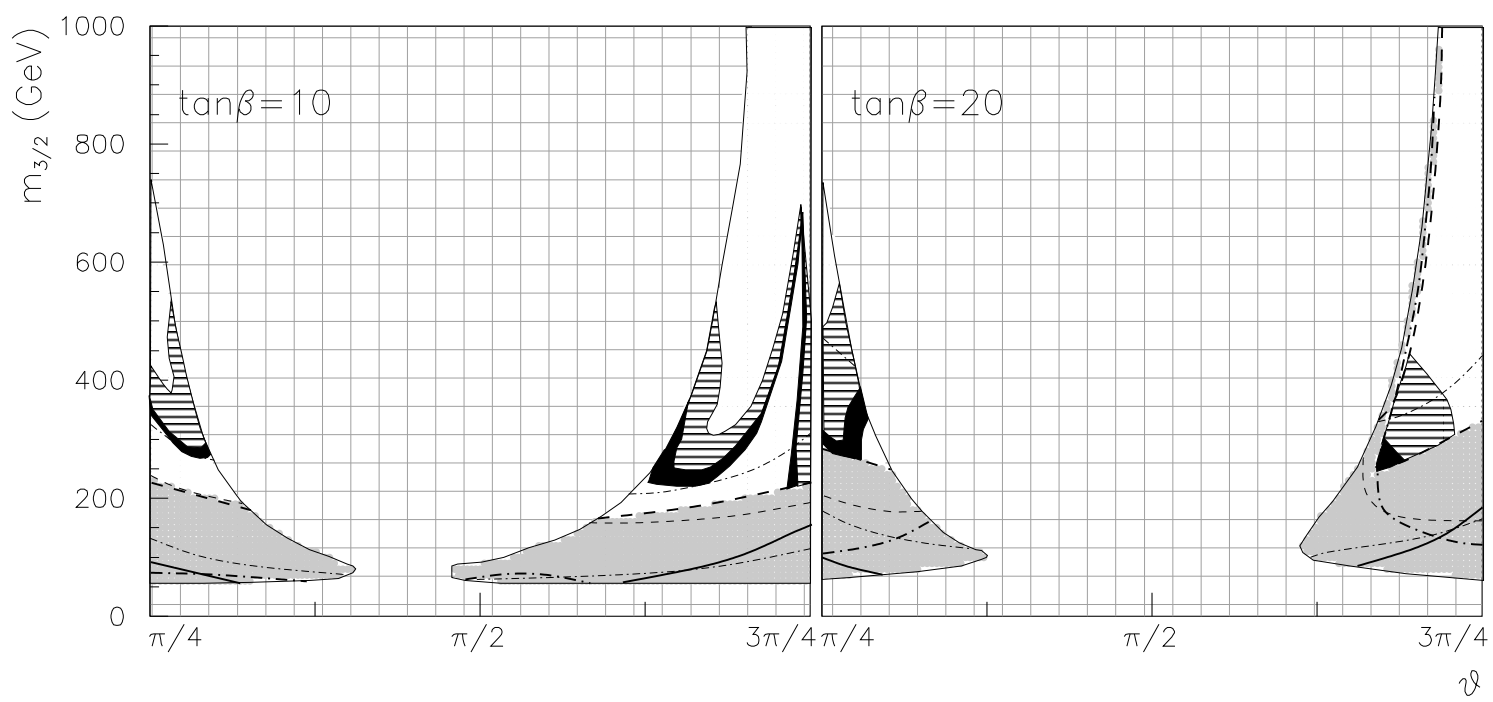

Figure 23: The same as Fig. 3 but for example A-III) of Table. 3 with $\tan \beta=10$ and 20. Soft masses satisfy (3.15) with slepton masses modified according to Eq.(4.30) and Higgs mass parameters given by (4.32).
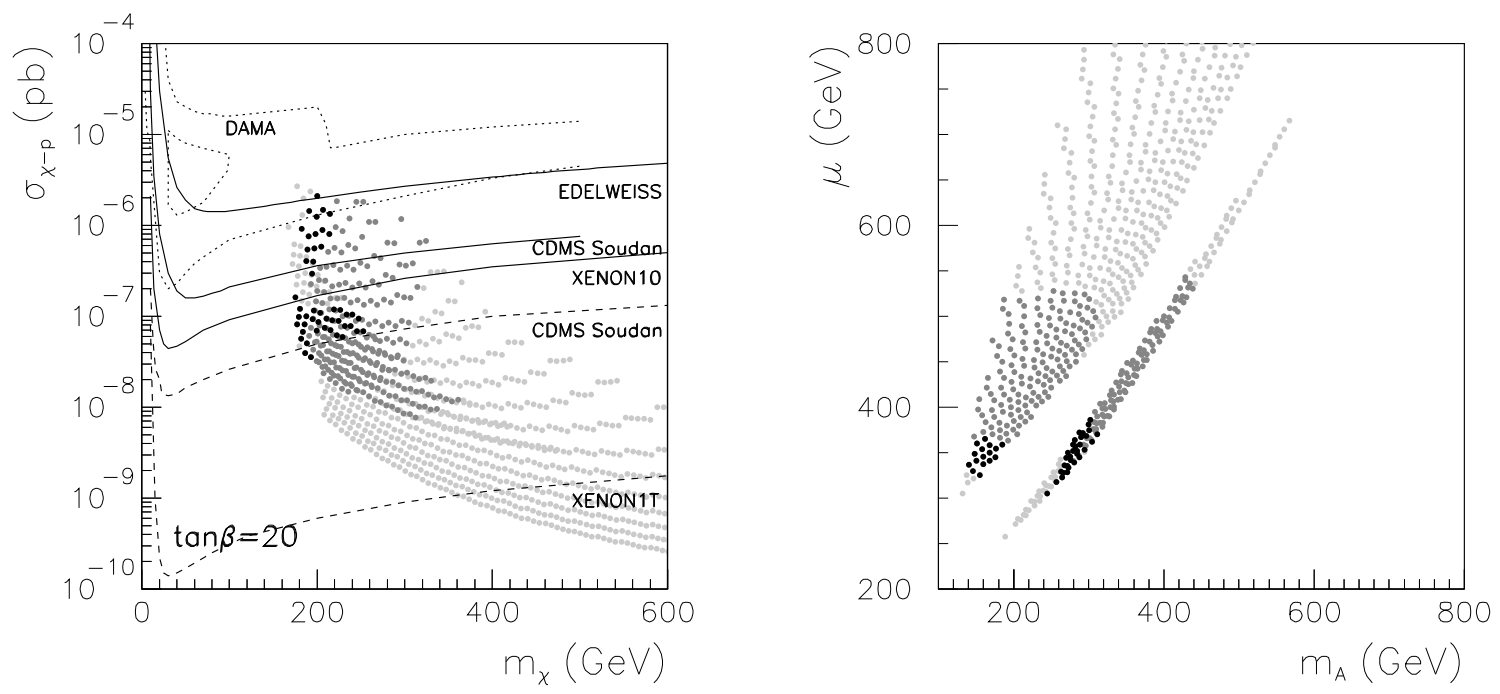

Figure 24: The same as Fig. 5 but for example A-III) of Table. 3 with $\tan \beta=20$. Soft masses satisfy (3.15) with slepton masses modified according to Eq.(4.30) and Higgs mass parameters given by (4.32).

$$
m_{H_{d}}^{2}=m_{3 / 2}^{2}\left(-4+5 \cos ^{2} \theta\right),
$$

there are only two narrow regions left with $200 \mathrm{GeV} \lesssim m_{3 / 2} \lesssim 400 \mathrm{GeV}$ around $\theta \approx 0.8$ and $\theta \approx 2.3$, for which $\delta_{L_{L}, e_{R}} \approx 0.8, \delta_{H_{u}} \approx 1.7, \delta_{H_{d}} \approx-2.6$, and $\delta_{L_{L}, e_{R}} \approx 1.6, \delta_{H_{u}} \approx 2.5$, 

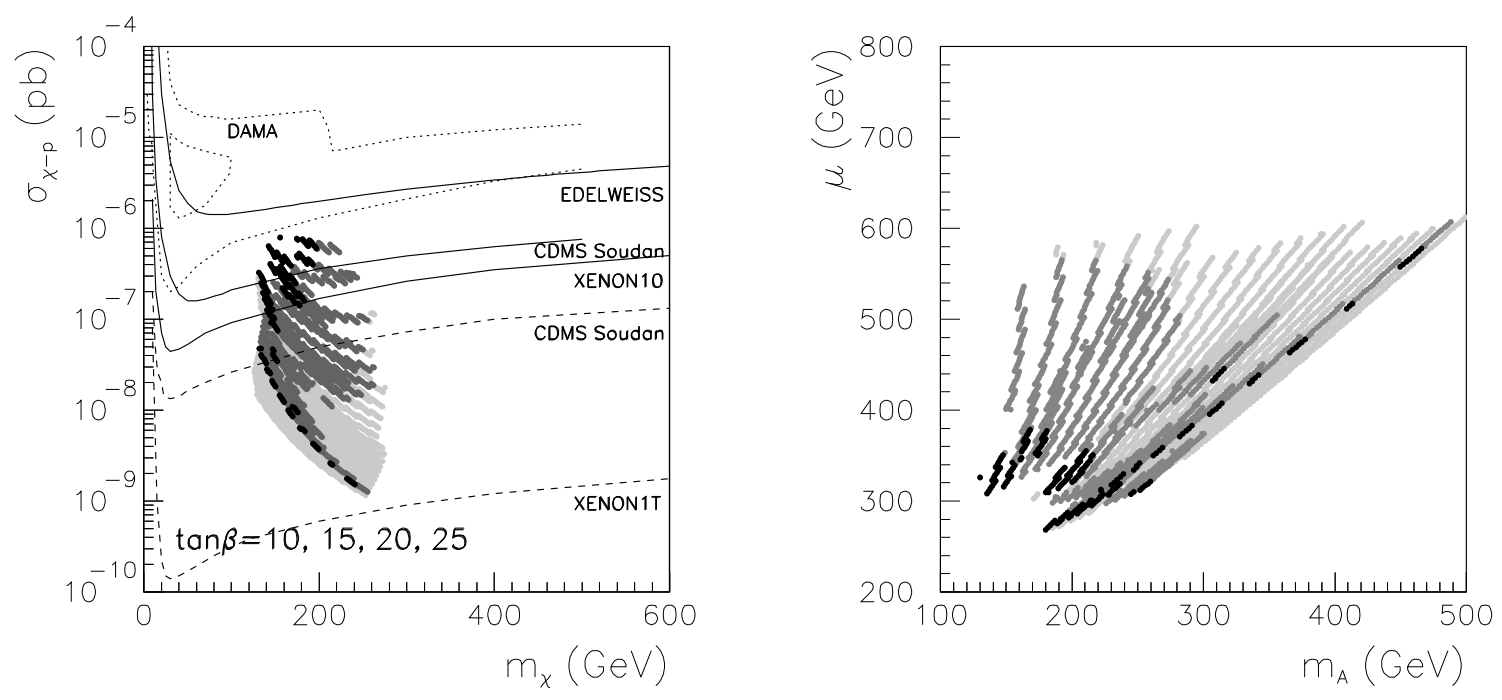

Figure 25: The same as Fig. 5 but for $\tan \beta=10,15,20$ and 25. Soft masses satisfy (3.15) and the effect of D-terms on the slepton and Higgs masses has been included according to Eqs.(4.30) and (4.33), respectively.

$\delta_{H_{d}} \approx-2.8$, respectively. The results for $\sigma_{\tilde{\chi}_{1}^{0}-p}$ are qualitatively similar to those of Fig. 24, with points within the reach of CDMS Soudan and XENON10 for $\tan \beta=10$ to 25 . The complete $\left(m_{3 / 2}, \theta\right)$ plane is ruled out for $\tan \beta \gtrsim 25$ due to the smallness of $m_{A}^{2}$.

In order to illustrate this possibility, the theoretical predictions for $\sigma_{\tilde{\chi}_{1}^{0}-p}$ are represented in Fig. 25 for a scan of the cases with $\tan \beta=10,15,20$ and 25. The neutralino detection cross section reaches the sensitivity of the CDMS Soudan and XENON10 experiments for $m_{\tilde{\chi}_{1}^{0}} \approx 150-200 \mathrm{GeV}$. On the right-hand side of Fig. 25 the corresponding values of $\mu$ are plotted as a function of the CP-odd Higgs mass, evidencing the important decrease in both as a consequence of Higgs non-universalities.

If the ratio of $U(1)$ charges is further increased to $q_{H_{d}}^{A} / q_{C}^{A}=2$, the whole parameter space becomes excluded.

Notice finally that, although we have exemplified the relevance of the contribution due to an anomalous $U(1)$ with a variation of case A) of Table1, the rest of the examples in that table can also improve their behaviour under UFB constraints and lead to detectable neutralino dark matter by using this procedure. This owes to the fact that the non-universality introduced by the D-term contribution typically dominates over the modular weights, especially in the dilaton limit, as we noted in Eq. (4.28). 


\section{Conclusions}

We have studied the phenomenology of supergravity theories which result from orbifold scenarios of the heterotic superstring and the resulting theoretical predictions for the direct detection of neutralino dark matter. These scenarios are specially interesting, since the soft terms can be computed explicitly and have, in general, a non-universal structure.

We have studied the parameter space of these constructions, computing the lowenergy spectrum and taking into account the most recent experimental and astrophysical constraints. In addition, we have imposed the absence of dangerous charge and colour breaking minima. In the remaining allowed regions the spin-independent part of the neutralino-proton cross section has been calculated and compared with the sensitivity of current and projected dark matter detectors.

In the absence of an anomalous $U(1)$ the non-universality of the soft scalar mass parameters is always negative with respect to the gravitino mass. The smallness of the stau mass implies that the UFB-3 direction in the parameter space becomes very deep and the realistic minimum is no longer the global one. In most of the cases it is not possible to satisfy this constraint in the regions permitted by astrophysical and experimental bounds. Also, the non-universality on the Higgs masses is not sufficient to produce any significant increase of the neutralino-nucleon cross section. Consequently, the theoretical predictions for $\sigma_{\tilde{\chi}_{1}^{0}-p}$ are typically beyond the sensitivity of dark matter experiments.

The presence of an anomalous $U(1)$ gives more flexibility to the non-universality in the scalar masses. These can even be heavier than the gravitino mass at the GUT scale. This allows to increase the slepton masses, thus avoiding the UFB constraints. Moreover, the lightest neutralino becomes the LSP in most of the parameter space and regions fulfilling all the experimental and astrophysical constraints can be found. Furthermore, the non-universality of the Higgs mass parameters can be tailored to favour the presence of light Higgses and the increase in the Higgsino composition of the lightest neutralino. This leads to a sizable increase in the theoretical predictions of $\sigma_{\tilde{\chi}_{1}^{0}-p}$ and compatibility with present experiments can be achieved.

\section{Acknowledgements}

D.G. Cerdeño is supported by the program "Juan de la Cierva" of the Ministerio de Educación y Ciencia of Spain. T. Kobayashi is supported in part by the Grand-in-Aid 
for Scientific Research \#17540251 and the Grant-in-Aid for the 21st Century COE "The Center for Diversity and Universality in Physics" from the Ministry of Education, Culture, Sports, Science and Technology of Japan. The work of C. Muñoz was supported in part by the Spanish DGI of the MEC under Proyecto Nacional FPA200605423, by the European Union under the RTN program MRTN-CT-2004-503369, and under the ENTApP Network of the ILIAS project RII3-CT-2004-506222. Likewise, the work of D. G. Cerdeño and C. Muñoz, was also supported in part by the Spanish DGI of the MEC under Proyecto Nacional FPA2006-01105, by the Comunidad de Madrid under Proyecto HEPHACOS, Ayudas de I+D S-0505/ESP-0346, and by the EU research and training network MRTN-CT-2006-035863.

\section{References}

[1] For a review, see C. Muñoz, 'Dark matter detection in the light of recent experimental results', Int. J. Mod. Phys. A 19 (2004) 3093 [arXiv:hep-ph/0309346].

[2] DAMA Collaboration, R. Bernabei et al., 'Search for WIMP annual modulation signature: results from DAMA/NaI-3 and DAMA/NaI-4 and the global combined analysis', Phys. Lett. B 480 (2000) 23; 'Dark matter search', Riv. Nuovo Cim. 26 (2003) 1 [arXiv:astro-ph/0307403].

[3] P. Belli, R. Cerulli, N. Fornengo and S. Scopel, 'Effect of the galactic halo modeling on the DAMA/NaI annual modulation result: an extended analysis of the data for WIMPs with a purely spin-independent coupling', Phys. Rev. D 66 (2002) 043503 [arXiv:hep-ph/0203242].

[4] CDMS Collaboration, D. S. Akerib et al.[CDMS Collaboration], 'First results from the cryogenic dark matter search in the Soudan underground lab', Phys. Rev. Lett. 93 (2004) 211301 [arXiv:astro-ph/0405033]; 'Limits on the spin-independent WIMP nucleon interactions from the two-tower run of the Cryogenic Dark Matter Search', Phys. Rev. Lett. 96 (2006) 011302 [arXiv:astro-ph/0509259].

[5] EDELWEISS Collaboration, A. Benoit et al., 'First results of the EDELWEISS WIMP search using a 320-g heat-and-ionization Ge detector', Phys. Lett. B 513 (2001) 15 [arXiv:astro-ph/0106094]; 'Improved exclusion limits from the 
EDELWEISS WIMP search', Phys. Lett.B 545 (2002) 43

[arXiv:astro-ph/0206271]; EDELWEISS Collaboration, V. Sanglard et al. 'Final results of the EDELWEISS-I dark matter search with cryogenic heat-and-ionization Ge detectors', Phys. Rev. D 71 (2005) 122002 [arXiv:astro-ph/0503265].

[6] UK Dark Matter Collaboration, G. J. Alner et al. 'First limits on nuclear recoil events from the ZEPLIN I galactic dark matter detector', Astropart. Phys. 23 (2005) 444.

[7] P. Ullio, M. Kamionkowski and P. Vogel, 'Spin-dependent WIMPs in DAMA?', JHEP 0107 (2001) 044 [arXiv:hep-ph/0010036];

D. Smith and N. Weiner, 'Inelastic dark matter', Phys. Rev. D 64 (2001) 043502 [arXiv:hep-ph/0101138]; 'Inelastic dark matter at DAMA, CDMS and future experiments', Nucl. Phys. Proc. Suppl. 124, 197 (2003) [arXiv:astro-ph/0208403]. R. Bernabei et al., 'Investigating the DAMA annual modulation data in the framework of inelastic dark matter', Eur. Phys. J. C 23 (2002) 61;

G. Prezeau, A. Kurylov, M. Kamionkowski and P. Vogel, 'New contribution to WIMP-nucleus scattering', Phys. Rev. Lett. 91 (2003) 231301 [arXiV:astro-ph/0309115];

C. Savage, P. Gondolo and K. Freese, 'Can WIMP spin dependent couplings explain DAMA data, in the light of null results from other experiments?', Phys. Rev. D 70 (2004) 123513 [arXiv:astro-ph/0408346].

[8] XENON Collaboration, J. Angle et al., 'First Results from the XENON10 Dark Matter Experiment at the Gran Sasso National Laboratory', arXiv:0706.0039 [astro-ph].

[9] R. Bernabei et al., 'Particle dark matter: From DAMA/NaI to DAMA/LIBRA', Phys. Atom. Nucl. 69 (2006) 2056; R. Bernabei et al., 'From DAMA/NaI to DAMA/LIBRA at LNGS', Eur. Phys. J. A 27 (2006) 57.

[10] J. Amaré et al., 'UZ dark matter searches at Canfranc', AIP Conf. Proc. 878 (2006) 99.

[11] See e.g., E. Aprile et al., 'The XENON dark matter search experiment', Nucl. Phys. Proc. Suppl. 138 (2005) 156 [arXiv:astro-ph/0407575]; 'The XENON dark matter experiment', arXiv:astro-ph/0502279. 
[12] D. N. Spergel et al., 'Wilkinson Microwave Anisotropy Probe (WMAP) three year results: implications for cosmology', Astrophys. J. Suppl. 170, 377 (2007) [arXiv:astro-ph/0603449]; C. L. Bennett et al., 'First year Wilkinson Microwave Anisotropy Probe (WMAP) observations: preliminary maps and basic results', Astrophys. J. Suppl. 148 (2003) 1 [arXiv:astro-ph/0302207];

[13] D. G. Cerdeño, E. Gabrielli, M. E. Gómez, and C. Muñoz, 'Neutralino nucleon cross section and charge and colour breaking constraints', JHEP 06 (2003) 030 [arXiv:hep-ph/0304115].

[14] K. Griest and L. Roszkowski, 'Effect of relaxing grand unification assumptions on neutralinos in the minimal supersymmetric model', Phys. Rev. D 46, 3309 (1992).

[15] V. Berezinsky, A. Bottino, J. Ellis, N. Fornengo, G. Mignola and S. Scopel, 'Neutralino dark matter in supersymmetric models with nonuniversal scalar mass terms', Astropart. Phys. 5 (1996) 1 [arXiv:hep-ph/9508249];

P. Nath and R. Arnowitt, 'Non-universal soft SUSY breaking and dark matter', Phys. Rev. D 56 (1997) 2820 [arXiv:hep-ph/9701301].

A. Corsetti and P. Nath, 'Gaugino mass nonuniversality and dark matter in sugra, strings and D-brane models', Phys. Rev. D 64 (2001) 125010 [arXiv:hep-ph/0003186].

[16] D. G. Cerdeño and C. Muñoz, 'Neutralino dark matter in supergravity theories with non-universal scalar and gaugino masses', JHEP 04 (2004) 015 [arXiv:hep-ph/0405057].

[17] S. Baek, Y. G. Kim and P. Ko, 'Neutralino dark matter scattering and $B_{s} \rightarrow \mu^{+} \mu^{-}$in SUSY models,' JHEP 02, 067 (2005) [arXiv:hep-ph/0406033].

[18] S. Baek, D. G. Cerdeño, Y. G. Kim, P. Ko and C. Muñoz, 'Direct detection of neutralino dark matter in supergravity', JHEP 06 (2005) 017 [arXiv:hep-ph/0505019].

[19] C. Muñoz, 'A kind of prediction from string phenomenology: extra matter at low energy', Mod. Phys. Lett. A 22, 989 (2007) [arXiv:0704.0987 [hep-ph]].

[20] For a review, see A. Brignole, L. E. Ibáñez and C. Muñoz, 'Soft supersymmetry-breaking terms from supergravity and superstring models', in 
the book 'Perspectives on Supersymmetry', Ed. G. Kane, World Scientific (1998) 125 [arXiv:hep-ph/9707209].

[21] S. Khalil, A. Masiero and Q. Shafi, 'From $b \rightarrow s \gamma$ to the LSP detection rates in minimal string unification models', Phys. Rev. D 56 (1997) 5754 [arXiv:hep-ph/9704234].

[22] M. Drees, Y. G. Kim, T. Kobayashi and M. M. Nojiri, 'Direct detection of neutralino dark matter and the anomalous dipole moment of the muon', Phys. Rev. D 63 (2001) 115009 [arXiv:hep-ph/0011359].

[23] P. Binétruy, Y. Mambrini and E Nezri, 'Direct and indirect detection of dark matter in heterotic orbifold models' Astropart. Phys. 22 (2004) 1 [arXiv:hep-ph/0312155].

[24] L. J. Dixon, J. Harvey, C. Vafa and E. Witten, 'Strings on orbifolds', Nucl. Phys. B 261 (1985) 678; 'Strings on orbifolds 2', Nucl. Phys. B 274 (1986) 285.

[25] L. E. Ibáñez, H. P. Nilles and F. Quevedo, 'Orbifolds and Wilson lines', Phys. Lett. B 187 (1987) 25.

[26] D. J. Gross, J. A. Harvey, E. J. Martinec and R. Rohm, 'The heterotic string', Phys. Rev. Lett 54 (1985) 502; 'Heterotic string theory. 1. The free heterotic string', Nucl. Phys. B 256 (1985) 253; 'Heterotic string theory. 2. The interacting heterotic string', Nucl. Phys. B 267 (1986) 75.

[27] L. E. Ibáñez, J. E. Kim, H. P. Nilles and F. Quevedo, 'Orbifolds compactifications with three families of $S U(3) \times S U(2) \times U(1)^{n}$, , Phys. Lett. B 191 (1987) 3.

[28] E. Witten, 'Some properties of O(32) superstrings', Phys. Lett. B 149 (1984) 351;

M. Dine, N. Seiberg and E. Witten, 'Fayet-Iliopoulos terms in string theory', Nucl. Phys. B 289 (1987) 317;

J. J. Atick, L. J. Dixon and A. Sen, 'String calculation of Fayet-Iliopoulos D terms in arbitrary supersymmetric compactifications', Nucl. Phys. B 292 (1987) 109 ;

M. Dine, I. Ichinose and N Seiberg, 'F terms and D terms in string theory', Nucl. Phys. B 293 (1987) 253. 
[29] J. A. Casas, E. K. Katehou and C. Muñoz, ' $U(1)$ charges in orbifolds: anomaly cancellation and phenomenological consequences', Nucl. Phys. B 317 (1989) 171.

[30] J. A. Casas and C. Muñoz, 'Three generation $S U(3) \times S U(2) \times U(1)_{Y} \times U(1)$ orbifold models through Fayet-Iliopoulos terms', Phys. Lett. B 209 (1988) 214.

[31] J. A. Casas and C. Muñoz, 'Three generation $S U(3) \times S U(2) \times U(1)_{Y}$ models from orbifolds', Phys. Lett. B 214 (1988) 63.

[32] A. Font, L. E. Ibáñez, H. P. Nilles and F. Quevedo, 'Yukawa couplings in degenerate orbifolds: towards a realistic $S U(3) \times S U(2) \times U(1)$ superstring', Phys. Lett. B 210 (1988) 101.

[33] J. Giedt, 'Spectra in standard-like $Z_{3}$ orbifold models', Annals Phys. 297 (2002) 67 [arXiv:hep-th/0108244].

[34] T. Kobayashi, S. Raby and R. J. Zhang, 'Constructing 5d orbifold grand unified theories from heterotic strings', Phys. Lett. B 593, 262 (2004) [arXiv:hep-ph/0403065]; 'Searching for realistic 4d string models with a Pati-Salam symmetry: Orbifold grand unified theories from heterotic string compactification on a $Z_{6}$ orbifold', Nucl. Phys. B 704 (2005) 3 [arXiv:hep-ph/0409098].

[35] W. Buchmuller, K. Hamaguchi, O. Lebedev and M. Ratz, 'Supersymmetric standard model from the heterotic string', Phys. Rev. Lett. 96 (2006) 121602 [arXiv:hep-ph/0511035]; 'Supersymmetric standard model from the heterotic string (II)', arXiv:hep-th/0606187.

[36] O. Lebedev, H. P. Nilles, S. Raby, S. Ramos-Sanchez, M. Ratz, P. K. S. Vaudrevange and A. Wingerter, 'A mini-landscape of exact MSSM spectra in heterotic orbifolds', Phys. Lett. B 645, 88 (2007) [arXiv:hep-th/0611095].

[37] J. E. Kim and B. Kyae, 'String MSSM through flipped SU(5) from $Z_{12}$ orbifold', arXiv:hep-th/0608085; 'Flipped SU(5) from $Z_{12-I}$ orbifold with Wilson line', Nucl. Phys. B 770, 47 (2007) [arXiv:hep-th/0608086].

[38] J. E. Kim, J. H. Kim and B. Kyae, 'Superstring standard model from $Z_{12-I}$ orbifold compactification with and without exotics, and effective R-parity', JHEP 0706, 034 (2007) [arXiv:hep-ph/0702278]. 
[39] H. Nakano, 'Scalar masses and cosmological constant induced by 'anomalous' U(1) D-term', Prog. Theor. Phys. Suppl. 123 (1996) 387 [arXiv:hep-ph/9404033].

[40] Y. Kawamura, 'Low-energy effective Lagrangian from non-minimal supergravity with unified gauge symmetry', Phys. Rev. D 53 (1996) 3779 [arXiv:hep-ph/9508290]; 'On low-energy theory from general supergravity', Prog. Theor. Phys. Suppl. 123 (1996) 421 [arXiv:hep-ph/9511334].

[41] Y. Kawamura and T. Kobayashi, 'Soft scalar masses in string models with anomalous $U(1)$ symmetry' Phys. Lett. B 375 (1996) 141 [arXiv:hep-ph/9601365], Erratum: 388867 (1996); 'Generic Formula of Soft Scalar Masses in String Models', Phys. Rev. D 56 (1997) 3844 [arXiv:hep-ph/9608233].

[42] Y. Kawamura, T. Kobayashi and T. Komatsu, 'Specific scalar mass relations in $S U(3) \times S U(2) \times U(1)$ orbifold model', Phys. Lett. B 400 (1997) 284 [arXiv:hep-ph/9609462].

[43] Y. Kawamura, 'Model-independent analysis of soft masses in heterotic string models with anomalous U(1) symmetry' Phys. Lett. B 446 (1999) 228 [arXiv:hep-ph/9811312].

[44] P. Binétruy and E. Dudas, 'Gaugino condensation and the anomalous $U(1)$ ', Phys. Lett. B 389 (1996) 503 [arXiv:hep-th/9607172];

G. R. Dvali and A. Pomarol, 'Anomalous $U(1)$ as a mediator of supersymmetry breaking', Phys. Rev. Lett. 77 (1996) 3728 [arXiv:hep-th/9607383];

N. Arkani-Hamed, M. Dine and S. P. Martin, 'Dinamical supersymmetry breaking in models with a Green-Schwarz mechanism', Phys. Lett. B 431 (1998) 329 [arXiv:hep-ph/9803432];

T. Barreiro, B. de Carlos, J. A. Casas and J. M. Moreno, 'Anomalous U(1), gaugino condensation and supergravity', Phys. Lett. B 445 (1998) 82 [arXiv:hep-ph/9808244];

E. Dudas and S. K. Vempati, 'Large D-terms, hierarchical soft spectra and moduli stabilisation', Nucl. Phys. B 727 (2005) 139 [arXiv:hep-th/0506172].

[45] P. Ko, T. Kobayashi, J. h. Park and S. Raby, 'String-derived D4 flavor symmetry and phenomenological implications,' arXiv:0704.2807 [hep-ph]. 
[46] J. A. Casas, A. Lleyda and C. Muñoz, 'Strong constraints on the parameter space of the MSSM from charge and colour breaking minima', Nucl. Phys. B 471 (1996) 3 [arXiv:hep-ph/9601357].

[47] A. Brignole, L. E. Ibáñez and C. Muñoz, 'Towards a theory of soft terms for the supersymmetric standard model', Nucl. Phys. B 422 (1994) 125, Erratum: 436 (1995) 747 [arXiv:hep-ph/9308271].

[48] T. Kobayashi, D. Suematsu, K. Yamada and Y. Yamagishi, 'Non-universal soft scalar masses in superstring theories', Phys. Lett. B 348 (1995) 402 [arXiv:hep-th/9408322].

[49] A. Brignole, L. E. Ibáñez, C. Muñoz and C. Scheich, 'Some issues in soft SUSY-breaking terms from dilaton/moduli sectors', Z. Phys. C 74 (1997) 157 [arXiv:hep-ph/9508258].

[50] L. E. Ibánez and D. Lüst, 'Duality anomaly cancellation, minimal string unification and the effective low-energy Lagrangian of 4-D strings' Nucl. Phys. B 382 (1992) 305 [arXiv:hep-th/9202046].

[51] V. Kaplunovsky and J. Louis, 'Model-independent analysis of soft terms in effective supergravity and in string theory' Phys. Lett. B 306 (1993) 269 [arXiv:hep-th/9303040].

[52] K. Choi, 'String unification and threshold effects', Phys. Rev. D 37 (1988) 1564.

[53] L. E. Ibáñez, D. Lüst and G. G. Ross, 'Gauge coupling running in minimal $S U(3) \times S U(2) \times U(1)$ superstring unification', Phys. Lett. B 272 (1991) 251 [arXiv:hep-th/9109053].

[54] Heavy Flavor Averaging Group (HFAG), E. Barberio et al., 'Averages of b-hadron properties at the end of 2005', arXiv:hep-ex/0603003; 'Averages of b-hadron properties at the end of 2006', arXiv:0704.3575 [hep-ex].

[55] CLEO Collaboration, S. Chen et al. 'Branching fraction and photon energy spectrum for $b \rightarrow s \gamma^{\prime}$, Phys. Rev. Lett. 87 (2001) 251807 [arXiv:hep-ex/0108032].

[56] BELLE Collaboration, P. Koppenburg et al., 'An inclusive measurement of the photon energy spectrum in $b \rightarrow s \gamma$ decays', Phys. Rev. Lett. 93 (2004) 061803 [arXiv:hep-ex/0403004]. 
[57] M. Misiak and M. Steinhauser, 'NNLO QCD corrections to the $\bar{B} \rightarrow X_{s} \gamma$ matrix elements using interpolation in $m_{c}{ }^{\prime}$, Nucl. Phys. B $\mathbf{7 6 4}$ (2007) 62 [arXiv:hep-ph/0609241]; M. Misiak et al., 'The first estimate of $\mathrm{B}\left(\bar{B} \rightarrow X_{s} \gamma\right)$ at $\mathcal{O}\left(\alpha_{s}^{2}\right)^{\prime}$, Phys. Rev. Lett. 98 (2007) 022002 [arXiv:hep-ph/0609232].

[58] CDF Collaboration, D. Acosta et al., 'Search for $B_{s}^{0} \rightarrow \mu^{+} \mu^{-}$and $B_{d}^{0} \rightarrow \mu^{+} \mu^{-}$ decays in $p \bar{p}$ collisions at $\sqrt{s}=1.96 \mathrm{TeV}$ ', Phys. Rev. Lett. 93 (2004) 032001 [arXiv:hep-ex/0403032].

[59] D0 Collaboration, V. M. Abazov et al. 'A search for the flavor-changing neutral current decay $B_{s}^{0} \rightarrow \mu^{+} \mu^{-}$in $p \bar{p}$ collisions at $\sqrt{s}=1.96 \mathrm{TeV}$ with the D0 detector', Phys. Rev. Lett. 94, 071802 (2005) [arXiv:hep-ex/0410039];

[60] R. Bernhard et al. 'A combination of CDF and D0 limits on the branching ratio of $B_{s(d)}^{0} \rightarrow \mu^{+} \mu^{-}$decays', arXiv:hep-ex/0508058.

[61] G. Bélanger, F. Boudjema, A. Pukhov and A. Semenov, 'micrOMEGAs: A program for calculating the relic density in the MSSM', Comput. Phys. Commun. 149 (2002) 103 [arXiv:hep-ph/0112278]; 'MicrOMEGAs: Version 1.3', Comput. Phys. Commun. 174 (2006) 577 [arXiv:hep-ph/0405253].

[62] Muon g-2 Collaboration, G. W. Bennett et al., 'Measurement of the negative muon anomalous magnetic moment to 0.7-ppm', Phys. Rev. Lett. 92, 161802 (2004) [arXiv:hep-ex/0401008].

[63] T. Kinoshita and W. J. Marciano, in Quantum Electrodynamics, ed. T. Kinoshita (World Scientific, Singapore, 1990), p. 419;

T. Kinoshita, 'New value of the $\alpha^{3}$ electron anomalous magnetic moment', Phys. Rev. Lett. 75 (1995) 4728;

A. Czarnecki, B. Krause and W. J. Marciano, 'Electroweak corrections to the muon anomalous magnetic moment', Phys. Rev. Lett. 76 (1996) 3267 [arXiv:hep-ph/9512369];

S. Eidelman and F. Jegerlehner, 'Hadronic contributions to $g-2$ of the leptons and to the effective fine structure constant $\alpha\left(M_{Z}^{2}\right)^{\prime}, Z$. Phys. C 67 (1995) 585 [arXiv:hep-ph/9502298];

K. Adel and F. J. Ynduráin, 'Improved evaluation of the hadronic vacuum polarization contributions to muon $g-2$ and $\bar{\alpha}_{\mathrm{QED}}\left(M_{Z}\right)$ using high order QCD calculations', arXiv:hep-ph/9509378; 
T. Kinoshita, B. Nizic and Y. Okamoto, 'Hadronic Contributions To The Anomalous Magnetic Moment Of The Muon', Phys. Rev. D 31 (1985) 2108;

J. Bijnens, E. Pallante and J. Prades, 'Hadronic light by light contributions to the muon $g-2$ in the large N(c) limit', Phys. Rev. Lett. 75 (1995) 1447 [Erratum-ibid. 75 (1995) 3781] [arXiv:hep-ph/9505251].

[64] M. Davier, S. Eidelman, A. Höcker and Z. Zhang, 'Updated estimate of the muon magnetic moment using revised results from $e^{+} e^{-}$annihilation', Eur. Phys. J. C 31 (2003) 503 [arXiv:hep-ph/0308213];

K. Hagiwara, A. D. Martin, D. Nomura and T. Teubner, 'Predictions for g-2 of the muon and $\alpha_{Q E D}\left(M_{Z}^{2}\right)^{\prime}$, Phys. Rev. D 69, 093003 (2004) [arXiv:hep-ph/0312250];

J. F. de Trocóniz and F. J. Ynduráin, 'The hadronic contributions to the anomalous magnetic moment of the muon', Phys. Rev. D 71, 073008 (2005) [arXiv:hep-ph/0402285].

[65] T. Kinoshita and M. Nio, 'The tenth-order QED contribution to the lepton $g-2$ : Evaluation of dominant $\alpha^{5}$ terms of muon $g-2$,' Phys. Rev. D 73 (2006) 053007 [arXiv:hep-ph/0512330];

K. Hagiwara, A. D. Martin, D. Nomura and T. Teubner, 'Improved predictions for $g-2$ of the muon and $\alpha_{Q E D}\left(M_{Z}^{2}\right)$, ' Phys. Lett. B 649 (2007) 173 [arXiv:hep-ph/0611102].

[66] J. A. Casas, A. Ibarra and C. Muñoz, 'Phenomenological viability of string and M-theory scenarios', Nucl. Phys. B 554 (1999) 67 [arXiv:hep-ph/9810266].

[67] M. Bolz, A. Brandenburg and W. Buchmuller, 'Thermal production of gravitinos', Nucl. Phys. B 606 (2001) 518 [arXiv:hep-ph/0012052].

[68] M. Kawasaki, K. Kohri and T. Moroi, 'Hadronic decay of the gravitino in the early universe and its implications to inflation', arXiv:hep-ph/0410287.

[69] I. Antoniadis, J. Ellis, S. Kelley and D. V. Nanopoulos, 'The price of deriving the standard model from string', Phys. Lett. B 272 (1991) 31;

I. Antoniadis, G. K. Leontaris and N. D. Tracas, 'Grand unified string models and low energy couplings', Phys. Lett. B 279 (1992) 58; 
D. Bailin and A. Love, 'String unification, grand unification and string loop threshold corrections', Phys. Lett. B 292 (1992) 315;

M. K. Gaillard and R. Xiu, 'Analysis of running coupling constant unification in string theory', Phys. Lett. B 296 (1992) 71;

A. E. Faraggi, 'Gauge coupling unification in superstring derived standard-like models', Phys. Lett. B 302 (1993) 202 [arXiv:hep-ph/9301268];

K. R. Dienes, A. E. Faraggi, 'Making ends meet: String unification and low-energy data', Phys. Rev. Lett. 75 (1995) 2646 [arXiv:hep-th/9505018]; 'Gauge coupling unification in realistic free fermionic string models', Nucl. Phys. B 457 (1995) 409 [arXiv:hep-th/9505046];

C. Bachas, C. Fabre and T. Yanagida, 'Natural gauge-coupling unification at the string scale' Phys. Lett. B 370 (1996) 49 [arXiv:hep-th/9510094];

D. Emmanuel-Costa, P. Fileviez-Perez and R. Gonzalez Felipe, 'Natural gauge and gravitational coupling unification and the superpartner masses' Phys. Lett. B 648 (2007) 60 [arXiv:hep-ph/0610178];

V. Barger, J. Jiang, P. Langacker and T. Li, 'String scale gauge coupling unification with vector-like exotics and non-canonical $U(1)_{Y}$ normalization', arXiv:hep-ph/0612206

[70] T. Kobayashi and H. Nakano, ' 'Anomalous' U(1) symmetry in orbifold string models', Nucl. Phys. B 496 (1997) 103 [arXiv:hep-th/9612066].

[71] J. Giedt, 'Completion of standard-like embeddings', Annals. Phys. 289 (2001) 251 [arXiv:hep-th/0009104].

[72] A. Ibarra, 'Charge and color breaking and D-terms in string theory', JHEP 01 (2002) 003 [arXiv:hep-ph/0111085]. 